\title{
Coadjoint orbit action of Virasoro group and two-dimensional quantum gravity dual to SYK/tensor models
}

\author{
Gautam Mandal, ${ }^{a}$ Pranjal Nayak $^{a}$ and Spenta R. Wadia ${ }^{b}$ \\ ${ }^{a}$ Department of Theoretical Physics, Tata Institute of Fundamental Research, \\ Mumbai 400005, India \\ ${ }^{b}$ International Centre for Theoretical Sciences, Tata Institute of Fundamental Research, \\ Shivakote, Bengaluru 560089, India \\ E-mail: mandal@theory.tifr.res.in, pranjal@theory.tifr.res.in, \\ spenta.wadia@icts.res.in
}

Abstract: The Nambu-Goldstone (NG) bosons of the SYK model are described by a coset space Diff $/ \mathrm{SL}(2, \mathbb{R})$, where Diff, or Virasoro group, is the group of diffeomorphisms of the time coordinate valued on the real line or a circle. It is known that the coadjoint orbit action of Diff naturally turns out to be the two-dimensional quantum gravity action of Polyakov without cosmological constant, in a certain gauge, in an asymptotically flat spacetime. Motivated by this observation, we explore Polyakov action with cosmological constant and boundary terms, and study the possibility of such a two-dimensional quantum gravity model being the AdS dual to the low energy (NG) sector of the SYK model. We find strong evidences for this duality: (a) the bulk action admits an exact family of asymptotically $\mathrm{AdS}_{2}$ spacetimes, parameterized by Diff $/ \mathrm{SL}(2, \mathbb{R})$, in addition to a fixed conformal factor of a simple functional form; (b) the bulk path integral reduces to a path integral over Diff $/ \mathrm{SL}(2, \mathbb{R})$ with a Schwarzian action; (c) the low temperature free energy qualitatively agrees with that of the SYK model. We show, up to quadratic order, how to couple an infinite series of bulk scalars to the Polyakov model and show that it reproduces the coupling of the higher modes of the SYK model with the NG bosons.
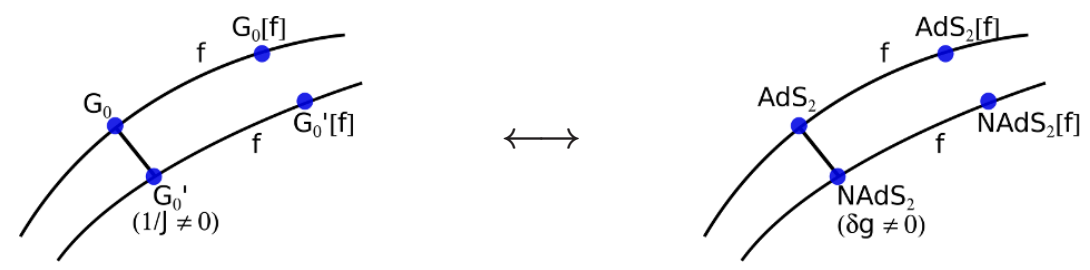

KeYwords: 2D Gravity, AdS-CFT Correspondence, Gauge-gravity correspondence ARXIV EPRINT: 1702.04266 


\section{Contents}

1 Introduction and summary 1

$\begin{array}{lll}2 & 2 \mathrm{D} \\ \text { quantum gravity action } & 4\end{array}$

2.1 Coadjoint orbits 5

$\begin{array}{lll}2.2 & \text { Two-dimensional quantum gravity action } & 6\end{array}$

3 Solutions of equations of motion and the Liouville action $\quad 8$

3.1 Equations of motion 8

$\begin{array}{ll}3.2 \text { Liouville action } & 10\end{array}$

4 Asymptotically $\mathbf{A d S}_{2}$ geometries $\quad 11$

4.1 Proper treatment of the bulk path integral 13

5 Action of hydrodynamics modes $\quad 13$

$\begin{array}{ll}5.1 \text { Boundary action } & 14\end{array}$

$\begin{array}{lll}5.2 & \text { Bulk action } & 16\end{array}$

$\begin{array}{lll}5.3 \text { Summary } & 18\end{array}$

6 Thermodynamic partition function from bulk dual 18

7 Comparison with field theory $\quad 21$

7.1 Hydrodynamics and a double scaling 21

$\begin{array}{lll}7.2 & \text { Thermodynamics } & 22\end{array}$

8 Discussion and open questions $\quad 22$

$\begin{array}{ll}\text { A Some identities } & 24\end{array}$

B Green's function of Laplacian in $\mathrm{AdS}_{2} \quad 24$

B.1 Green's function for thermal $\mathrm{AdS}_{2} 25$

C Variation of the induced gravity (Polyakov) action $\quad 26$

$\begin{array}{ll}\text { D Analysing off-shell constraints } & 32\end{array}$

E Exact computation of asymptotic $\mathrm{AdS}_{2}$ geometries $\quad 34$

F Quantum corrections to the classical action $\quad 35$

$\begin{array}{lll}\text { G Weyl anomaly in manifolds with boundary } & 37\end{array}$ 


\section{Introduction and summary}

The Sachdev-Ye-Kitaev (SYK) model and other tensor models that have universal IR properties [1-8], are quantum mechanical models of large $N$ fermionic particles, described by a Hamiltonian which, for Euclidean time $\tau=i t$, can be viewed alternatively as a onedimensional statistical model of fermions. The SYK model has random couplings $J_{i_{1} i_{2} \ldots i_{q}}$, representing disorder, and does not correspond to a unitary quantum mechanics. A different version without the random disorder, but with the same leading large $N$ behaviour, has been proposed by Gurau [6, 9], Witten [7], and Klebanov and Tarnopolsky [8]. Here we are interested only in the large $N$ behaviour and will call the set of models SYK-type models. More recently, higher dimensional generalizations of such models have also been a subject of study with the expectation that various interesting properties that make such models a good playground to study black hole physics can be carried over to the higher dimensions, [10-12].

SYK-type models have drawn a lot of attention in the literature recently (see, $[1,5-$ $8,10-23]$ for a partial list of related developments), primarily because of the following features in a large $N$ limit:

(1) There is an infrared fixed point with an emergent time reparametrisation symmetry, denoted henceforth as Diff..$^{1}$ The symmetry is spontaneously broken, at the IR fixed point, to $\mathbb{S L}(2, \mathbb{R})$ by the large $N$ classical solution, leading to Nambu-Goldstone (NG) bosons characterized by the coset Diff $/ \mathbb{S L}(2, \mathbb{R}){ }^{2}$ At the IR fixed point all these are precise zero modes of the action as one might expect from a one-dimensional CFT. Slightly away from the IR fixed point, the Diff symmetry is explicitly broken, the 'Nambu-Goldstone' modes cease to be zero modes and their dynamics is described by a Schwarzian term (which is the equivalent of a 'pion mass' term). It has been conjectured that (see, e.g. [24]) that this situation is similar to a bulk model in which the $\mathrm{AdS}_{2}$ symmetry is slightly broken (this is called a near $\mathrm{AdS}_{2}$ geometry, in the sense of an s-wave reduction from higher dimensions, as in [25]).

(2) The possibility of a gravity dual is further reinforced by the fact that the Lyapunov exponent in the SYK model saturates the chaos bound, which is characteristic of a theory of gravity that has black hole solutions [23, 26, 27].

(3) The full model has an approximately linearly rising ('Regge-type') spectrum of conformal weights near the IR fixed point, with $O(1)$ anomalous dimension even for operators with spin higher than two. This behaviour is unexpected both from string theory in the limit $\alpha^{\prime} \rightarrow 0$, or from Vasiliev theory (see, for example, [5]). Thus while the dynamics of the soft modes appears to have a simple dual gravity description, it is not clear if it can naturally incorporate the rest of the Regge-type spectrum description. In this paper we primarily concern ourselves with a bulk gravity dual which describes the soft modes. We leave the larger issue for later work.

\footnotetext{
${ }^{1}$ We use Diff to denote either $\operatorname{Diff}(R)$ or $\operatorname{Diff}\left(S^{1}\right)$, depending on whether we are at zero temperature or finite temperature. This group is alternatively called the Virasoro group.

${ }^{2}$ As explained later in more detail, unlike in higher dimensions where Nambu-Goldstone modes are zero modes of the action promoted to spacetime fields, here they remain zero modes (do not acquire kinetic terms) since they cannot be made dependent on any other dimension.
} 
The strategy we pursue for the proposed bulk dual is as follows.

As explained in $[1,5]$, the NG modes of the SYK-type model can be characterized by Diff orbits of the classical solution $G_{0}$ (at the IR fixed point $J=\infty$ ) or Diff orbits of $G_{0}^{\prime}$ which is the deformed value of $G_{0}$ after turning on a small value of $1 / J$ (see figure 1 ). Any given point on the Diff orbit can be obtained from the reference point, $G_{0}$ or $G_{0}^{\prime}$, by the action of an appropriate one-dimensional diffeomorphism.

It is shown in $[28,29]$ that the space of coadjoint orbits of Diff can be quantized using a natural symplectic form a la Kirillov [30], leading to Polyakov's two-dimensional quantum gravity action [31]. This observation is reminiscent of the emergent two-dimensional bulk description from the $c=1$ model, which is a matrix quantum mechanics. It was found in $[32,33]$ that the semiclassical (large $N$ ) singlet configurations of the matrix quantum mechanics, described by fermion droplets on a two-dimensional phase plane, could be understood as coadjoint orbits of $W_{\infty}$ algebra generated by bi-local boson operators made out of fermions. A representation of this algebra in $c=1$ was found in [34]. The coadjoint orbit action a la Kirillov [30] in the space of these configurations gave rise to a twodimensional action whose low energy sector reproduced the (massless) tachyons of twodimensional string theory. ${ }^{3}$ A similar approach was taken in [36] to arrive at a moduli space action of LLM geometries [37] describing half-BPS giant gravitons.

Following the above examples, one might wonder whether such a two-dimensional quantum gravity action, obtained by the coadjoint orbit method, naturally describes a bulk dual to the SYK model. It turns out that a priori it is not possible since the gravity action does not have a cosmological constant and it describes asymptotically flat spaces. This prompts us to consider a generalization of the Polyakov action, which includes a cosmological constant and boundary terms (the boundary terms are found by requiring the existence of a well-defined variational principle; these are also the terms required by consistency with the Weyl anomaly in a manifold with a boundary, see appendix G for details). The new action, described in section 2, has asymptotically $\mathrm{AdS}_{2}$ geometries as solutions (see section 4 and 3), which are all generated from $\mathrm{AdS}_{2}$ by the action of Diff. The schematics of these solutions is described in the right panel of figure 1.

The main point of the paper is that the two-dimensional quantum gravity theory, arrived at in this fashion, provides a bulk dual to the Nambu-Goldstone sector of the SYK models. We find a number of strong evidences for this duality:

(a) the space on which path integral of the bulk theory is performed reduces to $\operatorname{Diff} / \mathbb{S L}(2, \mathbb{R})$, which is the same as that of the Nambu-Goldstone bosons in the SYK model. In the bulk theory these degrees of freedom emerge as the space of large diffeomorphisms (analogous to Brown-Henneaux diffeomorphisms in $\mathrm{AdS}_{3}$ ). In addition to these, the bulk metric admits a fixed, non-dynamical conformal factor of a simple functional form. In the SYK theory this parameterizes the departure from strong coupling.

\footnotetext{
${ }^{3}$ The precise correspondence required some additional structure ('leg-poles'); see [35] for some recent insight.
} 

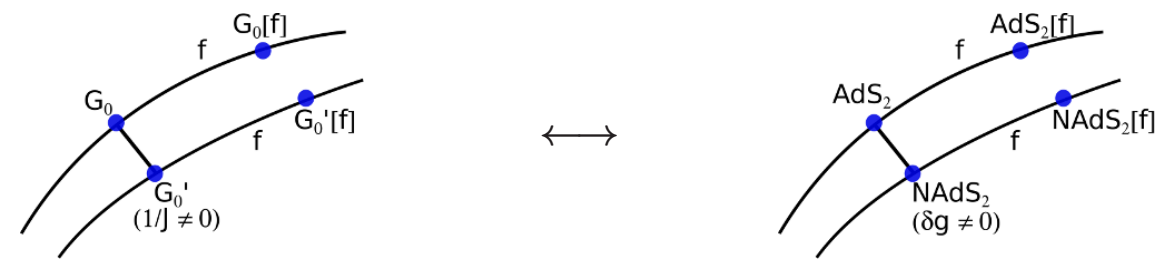

Figure 1. In the left panel, the top curve represents the $\operatorname{Diff}(R)$-orbit (or a $\operatorname{Diff}\left(S^{1}\right)$-orbit at finite temperature), at the IR fixed point $J=\infty$, of the classical large $N$ solution for the fermion bilocal $G_{0}\left(\tau_{1}, \tau_{2}\right) \sim\left(\tau_{1}-\tau_{2}\right)^{-2 \Delta}$; this represents the Nambu-Goldstones of $\operatorname{Diff}(R) / \mathbb{S L}(2, \mathbb{R})$. The lower curve represents the orbit of a deformed solution $G_{0}^{\prime}$ slightly away from the IR fixed point, with a small positive $1 / J$. In the right panel, the top curve represents the orbit of the $\mathrm{AdS}_{2}$ spacetime (these are asymptotically $\mathrm{AdS}_{2}$ spacetimes, the two-dimensional equivalent of Brown-Henneaux geometries, which we will describe explicitly in section 4). The bottom curve represents the orbit of a slightly deformed $\mathrm{AdS}_{2}$ spacetime $\mathrm{NAdS}_{2}$, with a controlled non-normalizable deformation (see section 4 ).

(b) The bulk path integral reduces to a path integral over Diff $/ \mathbb{S L}(2, \mathbb{R})$ with a Schwarzian action section 5 , characterized by a non-zero overall coefficient coming from the conformal factor.

(c) the low temperature free energy qualitatively agrees with that SYK model, section 5. In the Discussion section, we show how to go beyond the low energy sector, and describe the higher mass modes of the SYK model, by introducing bulk matter fields. We show, up to quadratic order, how to couple an infinite series of bulk scalars to the Polyakov model and show that it reproduces the coupling of the higher modes of the SYK model with the NG bosons.

Our paper is organized as follows. In section 2 we present a motivation for our proposed bulk action (2.7) from the viewpoint of coadjoint orbits of Diff $/ \mathbb{S L}(2, \mathbb{R})$. In the two subsequent sections, we analyze the theory in the conformal gauge $d s^{2}=e^{2 \phi} \widehat{d s^{2}}$. In section 3, we describe solutions of the equation of motion where $\widehat{d s^{2}}$ represents pure $\mathrm{AdS}_{2}$ geometry; it turns out that the 'Liouville mode' $\phi$ gets completely fixed by the equations of motion (in fact, by just the Virasoro constraints, as shown in appendix D), up to three real parameters which define boundary conditions for the metric. In section 4 we find a larger class of solutions, which represent large diffeomorphisms of $\mathrm{AdS}_{2}$ (similar to BrownHenneaux geometries in asymptotically $\mathrm{AdS}_{3}$ spacetimes). These are normalizable modes of the metric ('boundary gravitons') and represent dynamical variables of the path integral, which is described in section 4.1. In section 5 the effective action of these boundary gravitons is obtained by an on-shell evaluation of the path-integral; it is found to be given by a Schwarzian (5.17). Thus, the boundary gravitons are found to represent the pseudo-Nambu-Goldstone modes of the SYK model. In section 6, we focus on a large diffeomorphism which leads to a Euclidean black hole geometry (this turns the boundary direction into a circle). On-shell action for this geometry reproduces the qualitative features of the free energy of the SYK models. Detailed comparison with the SYK model is carried out in section 7. Finally, in section 8, we discuss how to describe the 'hard' modes of 
the SYK model in terms of external probe scalars coupled to the metric. The appendices contain detailed derivations of some formulae and supplementary arguments.

\section{$2 \quad 2 \mathrm{D}$ quantum gravity action}

In this section, we briefly review some of the material on coadjoint orbits of Diff in [28, $29,38]$, focussing on the emergence of $2 \mathrm{D}$ quantum gravity represented by the Polyakov action [31].

As explained in $[1,5]$, and briefly mentioned in the Introduction, the zero modes of the SYK model at the IR fixed point (we suggestively call these the Nambu-Goldstone (NG) modes, although they differ somewhat from their higher dimensional counterpart, as explained below) are given by Diff transforms of the large $N$ condensate of the bilocal 'meson' variable $G\left(\tau_{1}, \tau_{2}\right)=\psi_{I}\left(\tau_{1}\right) \psi_{I}\left(\tau_{2}\right),{ }^{4}$

$$
\begin{aligned}
G_{0}\left(\tau_{1}, \tau_{2}\right) & \sim \frac{1}{\left(\tau_{1}-\tau_{2}\right)^{2 \Delta}} \stackrel{f \in \operatorname{Diff}\left(R^{1}\right)}{\longrightarrow} G_{0}[f]\left(\tau_{1}, \tau_{2}\right) \\
G_{0}[f]\left(f\left(\tau_{1}\right), f\left(\tau_{2}\right)\right) & \equiv G_{0}\left(\tau_{1}, \tau_{2}\right)\left(\frac{\partial f\left(\tau_{1}\right)}{\partial \tau_{1}} \frac{\partial f\left(\tau_{2}\right)}{\partial \tau_{2}}\right)^{-\Delta}
\end{aligned}
$$

Here $f: \tau \rightarrow f(\tau)$ represents an element of $\operatorname{Diff}\left(R^{1}\right)$. This orbit is represented pictorially by the top curve in the left panel of figure 1 . In case of finite temperature, the time direction is considered Euclidean and compactified into a circle of size $\beta=1 / T$ : in that case the appropriate group of transformations is $\operatorname{Diff}\left(S^{1}\right)$.

The second line of the above equation essentially says that $G$ transforms as a bilocal tensor of weight $2 \Delta$ under the diffeomorphism $f$. For later reference, we give the infinitesimal version of this transformation as represented in the space of bilocal variables. (for $f(\tau)=\tau+\epsilon(\tau))$

$$
\delta_{\epsilon} G\left(\tau_{1}, \tau_{2}\right)=\left[\Delta\left(\partial_{\tau_{1}} \epsilon\left(\tau_{1}\right)+\partial_{\tau_{2}} \epsilon\left(\tau_{2}\right)\right)+\epsilon\left(\tau_{1}\right) \partial_{\tau_{1}}+\epsilon\left(\tau_{2}\right) \partial_{\tau_{2}}\right] G\left(\tau_{1}, \tau_{2}\right)
$$

Note that $G_{0}$, as defined in the first line, is invariant under $\mathbb{S L}(2, \mathbb{R})$, i.e. under Diff elements of the form $h(\tau)=(a \tau+b) /(c \tau+d)$, with $a d-b c=1$. This implies that the orbit described above parameterizes a coset Diff $/ \mathbb{S L}(2, \mathbb{R})$, namely the set of Diff elements quotiented by the identification $f(\tau) \sim f(h(\tau))$.

An important issue in the context of the SYK model is the quantum mechanical realization of the Diff algebra; in particular, it is an important question what the central charge of the corresponding Virasoro algebra is. We will find below, in terms of the bulk dual described by (2.7), that the central charge of the two-dimensional realization is proportional to $N .^{5}$

\footnotetext{
${ }^{4} \mathrm{We}$ are using a generalized notation here, in which ' $I$ ' denotes the appropriate indices of a given SYK/tensor model. For example, in SYK model it denotes the 'flavour indices' of fermions $\psi_{i}$, while in Witten-Gurau model it denotes the tri-fundamental index the fermions carry.

${ }^{5}$ More precisely, the Diff group is realized here as a subgroup of a two-dimensional conformal algebra which is unbroken by the presence of the boundary.
} 
In higher dimensions, such as in pion physics, the elements of the coset represent Nambu-Goldstone bosons, with kinetic terms given by a nonlinear sigma model (see, e.g. the discussion of pions in [39], chapter 19). The Nambu-Goldstone bosons are zero-modes promoted to spacetime-dependent fields. In the SYK model, the zero-modes are described by $f(\tau)$, or in the infinitesimal form $\epsilon(\tau),(2.2)$. Their definition already uses up the only dimension available in the model, and hence they cannot be made dependent on any other coordinate and remain zero modes (do not pick any kinetic terms). As explained in $[1,5]$, when we move away from the strict IR limit (i.e. when we turn on a small value of $1 / J$ ), these cease to be zero modes and pick up a non-zero action, given in terms of the Schwarzian derivative

$$
S_{\text {eff }} \sim \frac{N}{J} \int d \tau\{f, \tau\}, \text { where }\{f, \tau\} \equiv \frac{f^{\prime \prime \prime}(\tau)}{f^{\prime}(\tau)}-\frac{3}{2}\left(\frac{f^{\prime \prime}(\tau)}{f^{\prime}(\tau)}\right)^{2}
$$

In spite of the appearance of the derivatives, the above is a 'potential' term for the zero modes, similar to a pion mass term. ${ }^{6}$

\subsection{Coadjoint orbits}

The above discussion shows that the degrees of freedom of the low energy (NG) sector of the SYK theory are characterized by elements of $M=\operatorname{Diff} / \mathbb{S L}(2, \mathbb{R})$. In particular, the free energy is given by a path integral over $M$ with the above Schwarzian action.

In this subsection, we address the question of possible quantization of this configuration space. This question has a natural interpretation in terms of AdS/CFT correspondence, since the bulk path integral can, in a sense, be regarded as a radial quantum evolution of boundary data $[40-42] .^{7}$

The quantum theory envisaged above has a configuration space given by the group of paths in $M$ (the group of closed paths in $M$ is called loop $(M)$ ). An action functional on this space was formulated in [28, 29], using the formalism of coadjoint orbits and the resulting symplectic form in $M[30,38]$. Let us consider a path $\mathcal{P}(\sigma)$ in the space of Diff elements, with $\mathcal{P}(0)=P_{0}, \mathcal{P}(1)=P_{1}$. Since each point of the path is represented by a diffeomorphism, we can label the path as $f(\tau, \sigma)$ where the initial point $P_{0}$ corresponds to some diffeomorphism $f_{0}(\tau)$ and the final point $P_{1}$ to another diffeomorphism $f_{1}(\tau)$. The above mentioned action functional for such a path, also called the coadjoint orbit action or the Kirillov action, is given by $[28,29]$ (where the symplectic form is $\Omega=d \Theta$ )

$$
\begin{aligned}
S_{\text {Kirillov }} & =\int d \sigma \Theta(\sigma,\{f(\tau, \sigma)\}) \\
& =\int d \sigma d \tau\left[-b_{0}(\{f(\tau)\}) f^{\prime} \dot{f}+\frac{c}{48 \pi} \frac{f^{\prime}}{\dot{f}}\left(\frac{\dddot{f}}{\dot{f}}-2 \frac{\ddot{f}^{2}}{\dot{f}^{2}}\right)\right]
\end{aligned}
$$

\footnotetext{
${ }^{6}$ One way to appreciate this is to regard the Euclidean time as a discrete lattice and think of the 'time' derivatives in terms of discrete differences $f^{\prime}(\tau) \sim f_{i+1}-f_{i}$ where $f(\tau)$ is regarded as a collection of constant zero modes $f_{i}$.

${ }^{7}$ See [43] for a detailed treatment of the boundary wavefunction which represents the CFT data accurately.
} 
where $\dot{f}=\partial_{\tau} f, f^{\prime}=\partial_{\sigma} f$ etc. Here $c$ represents a possible central term in the coadjoint representation of Diff $[28,29,38] ; b_{0}$ is an arbitrary functional, representing the choice of a reference point on the orbit (different inequivalent orbits correspond to different inequivalent choices of $b_{0}$.)

It was observed in $[28,29]$ that, with the choice $b_{0}=0$ (we discuss this more later), the Kirillov action becomes the same as the two-dimensional quantum gravity action of Polyakov [31]

$$
S[g]=\frac{c}{24 \pi} \int_{\Gamma} \sqrt{g} R \frac{1}{\square} R
$$

where the metric is $[28]^{8}$

$$
d s^{2}=\partial_{\sigma} f d \tau d \sigma
$$

Here, $R$ is the Ricci scalar of the geometry, $\frac{1}{\square}$ is a notation used for the inverse of the scalar Laplacian in the geometry.

\subsection{Two-dimensional quantum gravity action}

It is rather remarkable that the two-dimensional quantum gravity action of Polyakov emerges from the quantization of the Diff configuration space. ${ }^{9}$ Identifying such a quantization with the holographic path integral, as mentioned in the previous subsection, one would tend to identify (2.5) with a possible bulk dual for the Nambu-Goldstone sector of the SYK model. This does not work, however, since the action (2.5) does not have a cosmological constant and therefore pertains to asymptotically flat spaces without a boundary. To qualify as the bulk dual, the classical action must admit asymptotically $\mathrm{AdS}_{2}$ spaces as solutions. Is there a natural generalization of the Polyakov action (2.5) which admits such solutions?

It turns out that there is such an action, given by ${ }^{10}$

$$
S_{\mathrm{cov}}[g]=\frac{1}{16 \pi b^{2}} \int_{\Gamma} \sqrt{g}\left[R \frac{1}{\square} R-16 \pi \mu\right]+\frac{1}{4 \pi b^{2}} \int_{\partial \Gamma} \sqrt{\gamma} \mathcal{K} \frac{1}{\square} R+\frac{1}{4 \pi b^{2}} \int_{\partial \Gamma} \sqrt{\gamma} \mathcal{K} \frac{1}{\square} \mathcal{K}
$$

Here $\mathcal{K}$ is the extrinsic curvature of the boundary. The constant $b^{2}=\frac{3}{2 c}$ is the dimensionless Newton's constant in two dimensions; we are interested in the classical limit $b \rightarrow 0$. A bulk cosmological constant, $(-\mu)<0,{ }^{11}$ is also included (to accommodate asymptotically $\mathrm{AdS}_{2}$ spaces). The boundary terms are dictated by the requirement of a well-defined variational principle (see appendix C for derivation); these terms can also be independently derived from the considerations of Weyl anomaly on manifolds with a boundary, see appendix G.

\footnotetext{
${ }^{8}$ The function $f(\tau, \sigma)$ here should be compared with $F(x, t)$ of [28].

${ }^{9}$ In the foregoing discussion, the fact that the Diff symmetry is slightly broken does not appear to be taken into account. Shortly we discuss how the broken Diff symmetry gets incorporated from the 2D gravity perspective.

${ }^{10}$ One might wonder whether other non-local terms like $\left(\frac{1}{\square} R\right)^{n}, n \in \mathbb{Z}^{+}$are allowed in the action. It can be shown that including such higher order terms in general leads to equations of motion that do not admit an asymptotically $\mathrm{AdS}_{2}$ spacetime.

${ }^{11}$ We have already incorporated a negative sign while writing the action, thus leaving $\mu>0$.
} 
We have presented a discussion of the quantum corrections contributing to the action in appendix F.

We propose that the modified quantum gravity action (2.7) describes a bulk dual of the low energy sector of the SYK model. In the rest of the paper, we present strong evidence in favour of this duality.

In the next section, we will discuss more details of the above action. We will discuss in the subsequent section the Diff orbit of $\mathrm{AdS}_{2}$ (asymptotically $\mathrm{AdS}_{2}$ metrics) in detail, and show that they are solutions of the equations of motion. We should note that the specific realization of this Diff orbit will differ somewhat from that of the above discussion. The most important difference is that in the above discussion (which assumes spacetime without a boundary) various points of the Diff orbit are actually diffeomorphic in 2D; in our construction below, the Diff orbits involve large diffeomorphisms in $2 D$ which are nontrivial near the boundary, and hence constitute physically distinguished configurations.

It is important to emphasize the following points:

1. The action (2.5) involves the dynamical variables $f(\tau, \sigma)$ representing the loop space $L(D i f f)$ (more precisely, $L(M), M=\operatorname{Diff} / \mathbb{S L}(2, \mathbb{R})$ ). It describes a quantization of $M$, which is different from simply integrating over $M$. The latter emerges in the description of the pseudo-Nambu-Goldstone modes of the SYK model. It is possible to identify the quantization of $M$ as the two-dimensional boundary dual to gravity on $\mathrm{AdS}_{3}$ (see, e.g., [44]). ${ }^{12}$

2. In this work, however, we consider a different variant of the model, namely (2.7), which, in addition to the term in (2.5) includes a negative cosmological constant and boundary terms, and consequently defines a theory of gravity in asymptotically $\mathrm{AdS}_{2}$ spaces.

3. As we will find, the only physical degrees of freedom of (2.7), reduce to $M$, parametrized by $f(\tau)$ (see, e.g. (4.7)) which lives on the boundary. The bulkboundary correspondence in this case essentially follows from two-dimensional diffeomorphism (this is somewhat reminiscent of Chern-Simon theories on a manifold with boundaries, or of $\mathrm{AdS}_{3} / \mathrm{CFT}_{2}$ duality). We will also find that the action describing the modes $f(\tau)$ is the Schwarzian action of SYK-type model and that the low temperature thermodynamics also have qualitative agreement with that of SYK.

4. We would like to emphasize that while (2.5), in the gauge (2.6), arises from a coadjoint orbit action of Diff, we do not yet have an explicit proof that our proposed bulk dual, described by (2.7), is also a coadjoint orbit action of Diff for asymptotically $\mathrm{AdS}_{2}$ geometries in some gauge. While this may eventually turn out to be true, the verification of our proposed duality in the rest of paper is independent of such a connection.

\footnotetext{
${ }^{12}$ We thank D. Stanford and E. Witten for illuminating correspondences on these points.
} 


\section{Solutions of equations of motion and the Liouville action}

In this section we will first discuss the equations of motion from the action (2.7). We will find that the solutions describe spacetimes of constant negative curvature, which include $\mathrm{AdS}_{2}$ as well as a three-parameter 'non-normalizable' deformation, which correspond to geometries whose boundary is displaced with respect to the original boundary of $\mathrm{AdS}_{2}$. We will subsequently discuss the on-shell action.

\subsection{Equations of motion}

We now discuss the solutions of the above action, (2.7). We relegate the details of the computations of the equations of motion to appendix $\mathrm{C}$ and summarize only the important results here. The equations of motion are,

$$
\begin{aligned}
0= & \frac{1}{16 \pi b^{2}}\left(g_{\mu \nu}(w)(2 R(w)+8 \pi \mu)+\int_{\Gamma}^{x}\left[-2 \nabla_{\mu}^{(w)} \nabla_{\nu}^{(w)} G(w, x) R(x)\right]\right. \\
& \left.+\int_{\Gamma}^{x} \int_{\Gamma}^{y}\left[\frac{\partial G(w, x)}{\partial w^{\mu}} \frac{\partial G(w, y)}{\partial w^{\mu}}-\frac{1}{2} g_{\mu \nu}(w) g^{\alpha \beta}(w) \frac{\partial G(w, x)}{\partial w^{\alpha}} \frac{\partial G(w, y)}{\partial w^{\beta}}\right] R(x) R(y)\right)
\end{aligned}
$$

It is more instructive to study the trace and traceless part of the equations separately, ${ }^{13}$

Trace part: $R(x)=-8 \pi \mu$

$$
\begin{aligned}
\text { Traceless part: } 0=\int_{\Gamma}^{x}\left[-2\left(\nabla_{\mu}^{(w)} \nabla_{\nu}^{(w)} G(w, x)-\frac{1}{2} g_{\mu \nu}(w) \square^{(w)} G(w, x)\right) R(x)\right] \\
+\int_{\Gamma}^{x} \int_{\Gamma}^{y}\left[\frac{\partial G(w, x)}{\partial w^{\mu}} \frac{\partial G(w, y)}{\partial w^{\mu}}-\frac{1}{2} g_{\mu \nu}(w) g^{\alpha \beta}(w) \frac{\partial G(w, x)}{\partial w^{\alpha}} \frac{\partial G(w, y)}{\partial w^{\beta}}\right] \\
\times R(x) R(y)
\end{aligned}
$$

Note that since $\mu>0$, the first equation, (3.2), signifies that the metric must have a constant negative curvature, which of course includes $\mathrm{AdS}_{2}$. Does $\mathrm{AdS}_{2}$ also satisfy (3.3)? What is the most general solution of both equations?

We will leave details to appendix $\mathrm{C}$, and state the main results here. Let us write the metric in conformal gauge around an $\mathrm{AdS}_{2}$ background, $g_{\alpha \beta}=e^{2 \phi} \hat{g}_{\alpha \beta}$, where

$$
\widehat{d s^{2}} \equiv \hat{g}_{\alpha \beta} d x^{\mu} d x^{\nu}=\frac{1}{\pi \mu(z+\bar{z})^{2}} d z d \bar{z}=\frac{1}{4 \pi \mu \zeta^{2}}\left(d \zeta^{2}+d \tau^{2}\right)
$$

Eq. (3.2) then becomes the same as Liouville equation of motion (see below for detail),

$$
2 \hat{\square} \phi=\hat{R}+8 \pi \mu e^{2 \phi}
$$

which has the general solution $[45,46]$,

$$
\phi=\frac{1}{2} \log \left[(z+\bar{z})^{2} \frac{\partial g(z) \bar{\partial} \bar{g}(\bar{z})}{(g(z)+\bar{g}(\bar{z}))^{2}}\right]
$$

\footnotetext{
${ }^{13}$ We will subsequently write the action, $(2.7)$ itself as sum over the trace and traceless part.
} 
where $g, \bar{g}$ are arbitrary complex functions, conjugate of each other. ${ }^{14}$ In the same gauge and background, (3.3) gives us the following Virasoro constraints,

$$
\partial^{2} \phi(z, \bar{z})-(\partial \phi(z, \bar{z}))^{2}+2 \frac{\partial \phi(z, \bar{z})}{z+\bar{z}}=0, \quad \bar{\partial}^{2} \phi(z, \bar{z})-(\bar{\partial} \phi(z, \bar{z}))^{2}+2 \frac{\bar{\partial} \phi(z, \bar{z})}{z+\bar{z}}=0
$$

Solving (3.2) and (3.3) (or, equivalently (3.7)) simultaneously, we get solutions (3.6) with following conditions on $g, \bar{g}$,

$$
\{g(z), z\}=0, \quad\{\bar{g}(\bar{z}), \bar{z}\}=0 \Rightarrow g(z)=\frac{a z+i b}{i c z+d}, \quad \bar{g}(\bar{z})=\frac{\bar{a} \bar{z}-i \bar{b}}{-i \bar{c} \bar{z}+\bar{d}}, \quad a, b, c, d \in \mathbb{C} .{ }^{15}
$$

Here, and subsequently in this paper we denote the Schwarzian derivative of a function, $f(\tau)$, by $\{f(\tau), \tau\}=\frac{f^{\prime \prime \prime}(\tau)}{f^{\prime}(\tau)}-\frac{3}{2}\left(\frac{f^{\prime \prime}(\tau)}{f^{\prime}(\tau)}\right)^{2}$. Of these solutions, the choice $a, b, c, d \in \mathbb{R}$ corresponds to $\mathbb{S L}(2, \mathbb{R})$ transformations of $\mathrm{AdS}_{2}$ coordinates, and are the exact isometries of the geometry.

The remaining 3-parameter set of solutions, which corresponds to the point marked $\mathrm{NAdS}_{2}$ in figure 1 are the solutions of our primary interest. These do not preserve the boundary of $\mathrm{AdS}_{2}$. In general, the boundary of the spacetime is given by the curve, $g(z)+\bar{g}(\bar{z})=0$, which for a general function of the kind, (3.8), is not the same as $z+\bar{z}=0$. These solutions will subsequently be referred to as non-normalizable solutions following the standard AdS/CFT language.

The set of non-normalizable solutions obtained above is clearly parameterized by $(a, b, c, d) \in \mathbb{S L}(2, \mathbb{C}) / \mathbb{S L}(2, \mathbb{R})$, which can be identified with a hyperboloid (see appendix $\mathrm{D}$, especially (D.7) for more details). The point $(a, b, c, d)=(1,0,0,1)$ corresponds to the identity transformation $g(z)=z$ in (3.8). We are interested in small non-normalizable deformations near the identity transformation. It is possible to choose a set of coordinates of $\mathbb{S L}(2, \mathbb{C}) / \mathbb{S L}(2, \mathbb{R})$, in which such deformations are given by

$$
a=1+i \delta a^{I} \quad b=i \delta b^{I} \quad c=i \delta c^{I} \quad d=1-i \delta a^{I},
$$

where $\delta a^{I}, \delta b^{I}, \delta c^{I}$ are real numbers. With these parameters, the solution for the metric becomes

$$
d s^{2}=e^{2 \phi} \widehat{d s^{2}}
$$

with $\widehat{d s^{2}}$ given by the $\mathrm{AdS}_{2}$ metric (3.4), and $\phi$, using (3.6) and (3.8) has the near-boundary form

$$
\phi=-\frac{\delta g(i \tau)}{\zeta}+\mathcal{O}\left(\delta a^{2}, \delta b^{2}, \delta c^{2}\right), \quad-\delta g(i \tau)=\delta b^{I}+2 \delta a^{I} \tau+\delta c^{I} \tau^{2}
$$

Eventually, we will choose $\delta a^{I}=\delta c^{I}=0$, so that $\delta g=-\delta b^{I}$, and $\phi=\delta b^{I} / \zeta$. We will find that the $\delta b^{I}$ deformation (more precisely, $-\delta b^{I}$ ) corresponds to the irrelevant coupling

\footnotetext{
${ }^{14}$ In Lorentzian signature, these functions can be chosen to be two independent real functions.

${ }^{15}$ Here the independent set of parameters are constrained by $a d+b c=1$, which is the same as $\mathbb{S L}(2, \mathbb{C})$.
} 
$1 / J$ of the SYK model. The other parameters $\delta a^{I}$ and $\delta c^{I}$ are physically distinct; it would be interesting to explore their significance, which we leave for future work.

For the Liouville factor $e^{2 \phi}$ not to destroy the asymptotic $\mathrm{AdS}_{2}$ structure altogether, we will assume here that $\delta g \lesssim \delta$; this ensures that $\delta g<\zeta .{ }^{16}$ Note that the expression for the Liouville field in (3.11) is similar to that of the dilaton in [24], and plays a somewhat similar role as we will see later. In the next section, we will generate more solutions from the above three-parameter solutions by using large diffeomorphisms, which we cannot capture staying within the conformal gauge.

\subsection{Liouville action}

We now show that the above analysis of equations of motion with separation into trace and traceless parts also works for the classical action. Writing the induced gravity action in a conformal gauge around an arbitrary fiducial metric, $\hat{g}_{\alpha \beta}$, we get the action, ${ }^{17}$

$$
\begin{aligned}
S_{\text {cov }}[g]= & -\frac{1}{4 \pi b^{2}}\left[\int_{\Gamma} \sqrt{\hat{g}}\left(\hat{g}^{\alpha \beta} \partial_{\alpha} \phi \partial_{\beta} \phi+\hat{R} \phi+4 \pi \mu e^{2 \phi}\right)+2 \int_{\partial \Gamma} \sqrt{\hat{\gamma}} \hat{\mathcal{K}} \phi+\int_{\partial \Gamma} \sqrt{\hat{\gamma}} \hat{n}^{\mu} \phi \partial_{\mu} \phi\right. \\
& \left.-\frac{1}{2} \int_{\partial \Gamma} \sqrt{\hat{\gamma}} \hat{n}^{\mu} \hat{\nabla} \mu\left(\phi \frac{1}{\hat{\square}} \hat{R}\right)\right]+\frac{1}{16 \pi b^{2}} \int_{\Gamma} \sqrt{\hat{g}} \hat{R} \frac{1}{\hat{\square}} \hat{R}+\frac{1}{4 \pi b^{2}} \int_{\partial \Gamma} \sqrt{\gamma} \hat{\mathcal{K}} \frac{1}{\hat{\square}} \hat{R} \\
= & -\frac{1}{4 \pi b^{2}}\left[\int_{\Gamma} \sqrt{\hat{g}}\left(\hat{g}^{\alpha \beta} \partial_{\alpha} \phi \partial_{\beta} \phi+\hat{R} \phi+4 \pi \mu e^{2 \phi}\right)+2 \int_{\partial \Gamma} \sqrt{\hat{\gamma}} \hat{\mathcal{K}} \phi+\int_{\partial \Gamma} \sqrt{\hat{\gamma}} \hat{n}^{\mu} \phi \partial_{\mu} \phi\right] \\
& +\frac{1}{16 \pi b^{2}} \int_{\Gamma} \sqrt{\hat{g}} \hat{R} \frac{1}{\hat{b}} \hat{R}
\end{aligned}
$$

In all the above equations, the coordinate dependence of the functions is understood. In the second line above, we have dropped the terms boundary terms containing the Green's function, $\frac{1}{\tilde{\Lambda}}$, given the fall-off properties of the Green's function. We identify the part of the action in (3.12) which depends on $\phi$ field with Liouville action on a background with metric $\hat{g}$.

$$
S_{L}[\phi, \hat{g}]=-\frac{1}{4 \pi b^{2}}\left[\int_{\Gamma} \sqrt{\hat{g}}\left(\hat{g}^{\alpha \beta} \partial_{\alpha} \phi \partial_{\beta} \phi+\hat{R} \phi+4 \pi \mu e^{2 \phi}\right)+2 \int_{\partial \Gamma} \sqrt{\hat{\gamma}} \hat{\mathcal{K}} \phi+\int_{\partial \Gamma} \sqrt{\hat{\gamma}} \hat{n}^{\mu} \phi \partial_{\mu} \phi\right]
$$

We are interested in computing the above action in the classical limit, $b \rightarrow 0$. The classical equation of motion for the $\phi$ field turns out to be exactly the same as (3.5), the trace part of the equations of motion coming from the Polyakov action, as expected. We emphasize the fact that if one chooses to study (3.12) as a theory of quantum gravity, then the trace of background metric appearing there should not be treated as independent degree of freedom.

\footnotetext{
${ }^{16}$ There is a natural RG interpretation of this inequality in terms of the boundary theory. We will later identify $\delta g$ with $\sim 1 / J$ (see $(7.3)$ ). Together with the natural identification of $1 / \zeta$, for small $\zeta$, with a Wilsonian floating cut-off $\Lambda$ (to be distinguished from the bare cut-off $\Lambda_{0}=1 / \delta$, see [41, 42], also [43]), we find $\delta g / \zeta \sim \Lambda / J=1 / \bar{J}$, where $\bar{J}=J / \Lambda$ is the dimensionless coupling. Since $\bar{J}$ grows large near the IR cut-off, it follows that $\delta g / \zeta \ll 1$ near the IR cut-off.

${ }^{17}$ Later in this paper we will choose the fiducial metric from a class of Asymptotic $\mathrm{AdS}_{2}\left(\mathrm{AAdS}_{2}\right)$ geometries. Although none of the analysis depends on the choice of this fiducial metric, it is only economical for a classical analysis that we choose it to be one of the saddle point solutions.
} 
One-dimensional Liouville equation of motion has appeared in $[13,18,47]$ in the context of boundary dynamics. However, their connection to the induced gravity action that we have discussed here is not clear.

No dynamical Liouville mode. It is important to note that in our problem there are no dynamical Liouville modes at all. The Liouville mode is entirely fixed in terms of three parameters which, furthermore, correspond to non-normalizable modes. These are specified as boundary conditions of the path integral and are not dynamical variables. We elaborate on this point further in appendix D where we show that the form of the Liouville mode, with three real constants, is completely fixed by the Virasoro constraints alone.

\section{Asymptotically $\mathrm{AdS}_{2}$ geometries}

In this section, we will construct asymptotically $\mathrm{AdS}_{2}$ geometries as a Diff orbit of the solutions constructed in (3.10) (see the orbits in the right panel of figure 1). To begin with, we will construct these asymptotic geometries purely kinematically, from an analysis of asymptotic Killing vectors (AKV) of $\mathrm{AdS}_{2}$ geometry (also see appendix E for some details). Later, we argue that they solve the equations of motion and evaluate the onshell action for these configurations. AKV's of $\mathrm{AdS}_{2}$ have been studied earlier in [48, 49] in the near-boundary region, inspired by earlier work of Brown and Henneaux in one higher dimension [50]. We show below that it is possible to integrate the infinitesimal diffeomorphisms exactly to find the full nonlinear solution. This will lead to a class of $\mathrm{AAdS}_{2}$ geometries that are related to each other by diffeomorphisms that become tangential at the boundary. These geometries are dual to the conformally transformed states in the 1-D field theory. ${ }^{18}$ We mainly consider Euclidean metrics below.

Euclidean $\mathrm{AdS}_{2}$ metric in Poincare coordinates is defined by (3.4). The $\mathrm{AAdS}_{2}$ geometries are defined by the fall-off conditions [48-50],

$$
g_{\zeta \zeta}=\frac{1}{4 \pi \mu \zeta^{2}}+\mathcal{O}\left(\zeta^{0}\right), \quad g_{\zeta \tau}=\mathcal{O}\left(\zeta^{0}\right), \quad g_{\tau \tau}=\frac{1}{4 \pi \mu \zeta^{2}}+\mathcal{O}\left(\zeta^{0}\right)
$$

Variation of the metric under most general diffeomorphism is,

$$
\delta g_{\alpha \beta}=\nabla_{\alpha} \epsilon_{\beta}+\nabla_{\beta} \epsilon_{\alpha}=\left(\begin{array}{cc}
-\frac{\epsilon^{\zeta}(\zeta, \tau)-\zeta \partial_{\zeta} \epsilon^{\zeta}(\zeta, \tau)}{2 \pi \mu \zeta^{3}} & \frac{\partial_{\tau} \epsilon^{\zeta}(\zeta, \tau)+\partial_{\zeta} \epsilon^{\tau}(\zeta, \tau)}{4 \pi \mu \zeta^{2}} \\
\frac{\partial_{\tau} \epsilon^{\zeta}(\zeta, \tau)+\partial_{\zeta} \epsilon^{\tau}(\zeta, \tau)}{4 \pi \mu \zeta^{2}} & -\frac{\epsilon^{\zeta}(\zeta, \tau)-\zeta \partial_{\tau} \epsilon^{\tau}(\zeta, \tau)}{2 \pi \mu \zeta^{3}}
\end{array}\right)
$$

The asymptotic Killing vectors can be solved for by imposing on (4.2) the fall-off conditions in (4.1), [48, 49]. However, we choose to work in Fefferman-Graham gauge which is defined by,

$$
\delta g_{\zeta \zeta}=0, \quad \delta g_{\zeta \tau}=0
$$

\footnotetext{
${ }^{18}$ As indicated before, precisely at the conformal point, the stress tensor vanishes trivially; hence all states are ground states. However, slightly away from the conformal point, the (broken) conformal transformations lead to nontrivial states.
} 
The solution for the asymptotic Killing vectors is given in terms of an arbitrary function, $\delta f(\tau)$,

$$
\epsilon^{\zeta}(\zeta, \tau)=\zeta \delta f^{\prime}(\tau), \quad \epsilon^{\tau}(\zeta, \tau)=\delta f(\tau)-\frac{1}{2} \zeta^{2} \delta f^{\prime \prime}(\tau)
$$

It is clear from the above solution, that the diffeomorphism is tangential at the boundary of $\mathrm{AdS}_{2}, \zeta=0$. The integrated form of the coordinate transformations is,

$$
\tilde{\tau}=f(\tau)-\frac{2 \zeta^{2} f^{\prime \prime}(\tau) f^{\prime}(\tau)^{2}}{4 f^{\prime}(\tau)^{2}+\zeta^{2} f^{\prime \prime}(\tau)^{2}}, \quad \tilde{\zeta}=\frac{4 \zeta f^{\prime}(\tau)^{3}}{4 f^{\prime}(\tau)^{2}+\zeta^{2} f^{\prime \prime}(\tau)^{2}}
$$

Although we think that this choice of gauge should not be necessary and it should be possible to integrate the diffeomorphisms more generally, we found it easier to do so with this gauge choice. This was largely motivated by $[51,52]$ who performed similar integrations of diffeomorphisms in $\mathrm{AdS}_{3}$ case. The details of this computation are presented in appendix E.

The result of this diffeomorphism can be stated as follows. If we start with the $\mathrm{AdS}_{2}$ metric in the $\tilde{\zeta}-\tilde{\tau}$ coordinates

$$
\widehat{d s^{2}}=\frac{1}{4 \pi \mu \tilde{\zeta}^{2}}\left(d \tilde{\zeta}^{2}+d \tilde{\tau}^{2}\right)
$$

in the original $\zeta-\tau$ coordinates it becomes

$$
\widehat{d s^{2}}=\frac{1}{4 \pi \mu \zeta^{2}}\left(d \zeta^{2}+d \tau^{2}\left(1-\zeta^{2} \frac{\{f(\tau), \tau\}}{2}\right)^{2}\right)
$$

Recall that $\{f(\tau), \tau\}=\frac{f^{\prime \prime \prime}(\tau)}{f^{\prime}(\tau)}-\frac{3}{2}\left(\frac{f^{\prime \prime}(\tau)}{f^{\prime}(\tau)}\right)^{2}$ is the standard notation for Schwarzian derivative that we use throughout this paper. We want to emphasize that the class of geometries given by (4.6) also have constant negative curvature, $\hat{R}=-8 \pi \mu$. As in $\mathrm{AdS}_{3}$, it should be possible to identify these geometries as different sections of the global $\mathrm{AdS}_{2}$ geometry. Some discussion of how various $\mathrm{AdS}_{2}$ geometries are related is provided in [49].

One can carry out the above diffeomorphism in the presence of the non-normalizable solutions described in the previous section. To do this, we begin with the metric (3.10) in the $\tilde{\zeta}-\tilde{\tau}$ coordinates:

$$
d s^{2}=e^{2 \tilde{\phi}\left(\tilde{x}^{\mu}\right)} \widehat{d s^{2}}, \quad \tilde{\phi}\left(\tilde{x}^{\mu}\right)=\frac{\tilde{\delta g}}{\tilde{\zeta}}+\mathcal{O}\left(\delta a^{2}, \delta b^{2}, \delta c^{2}\right), \tilde{\delta g}=\operatorname{Im}(\delta b)+2 \operatorname{Im}(\delta a) \tilde{\tau}+\operatorname{Im}(\delta c) \tilde{\tau}^{2}
$$

and transform to $\zeta-\tau$ coordinates, yielding the metric

$$
\begin{aligned}
d s^{2} & =e^{2 \phi} \widehat{d s^{2}}, \widehat{d s^{2}}=\frac{1}{4 \pi \mu \zeta^{2}}\left(d \zeta^{2}+d \tau^{2}\left(1-\zeta^{2} \frac{\{f(\tau), \tau\}}{2}\right)^{2}\right) \\
\phi & =-\frac{\delta g(i \tilde{\tau})}{\tilde{\zeta}(\zeta, \tau)}+\mathcal{O}\left(\delta a^{2}, \delta b^{2}, \delta c^{2}\right),-\delta g(i \tilde{\tau})=\delta b^{I}+2 \delta a^{I} \tilde{\tau}+\delta c^{I} \tilde{\tau}^{2}, \tilde{\tau}=f(\tau)
\end{aligned}
$$

In terms of the figure 1, the above solutions (4.6), (4.7) represent the Diff orbit of AdS 2 and $N A d S_{2}$ on the right panel. 
As remarked below (3.11), we eventually choose only the one-parameter deformation parameterized by $\delta b^{I}$, which will turn out to correspond to the $1 / J$ deformation of the strong coupling fixed point of the SYK theory. However, for the sake of generality, we will for now continue with the more general form of $\delta g$.

\subsection{Proper treatment of the bulk path integral}

To this point we have not discussed the issue of gauge fixing inside the quantum mechanical path integral. While we are largely interested in a classical computation in the bulk, where the path integral measure due to gauge fixing is not important, we now shed some light on this issue. The computation of the ghost action is discussed in detail in appendix F. The gauge fixing $\delta$-function and the corresponding Faddeev-Popov determinant is given by,

$$
\begin{aligned}
1= & \Delta_{\mathrm{FP}}[\hat{g}[f(\tau)], \phi] \times \int\left[\mathcal{D} \epsilon^{(s)}\right][\mathcal{D} \phi][\mathcal{D} f(\tau)] \delta\left(g^{\epsilon^{(s)}}-e^{2 \phi} \hat{g}[f(\tau)]\right) \\
& \times \delta\left(\epsilon^{(s)}\left(z_{1}\right)\right) \delta\left(\epsilon^{(s)}\left(z_{2}\right)\right) \delta\left(\epsilon^{(s)}\left(z_{3}\right)\right)
\end{aligned}
$$

In line with the discussion of the previous sections, we gauge fix an arbitrary metric to be conformally related to the $\mathrm{AAdS}_{2}$ metrics. In the choice of this gauge, there is an additional $\mathbb{S L}(2, \mathbb{R})$ residual gauge freedom that has been fixed using the $\delta$-functions that anchor three arbitrary points in the geometry. ${ }^{19}$ Going through the standard procedure of introducing the fermionic ghosts, we obtain a ghost action (F.3). This procedure should not only capture the correct Jacobian required for the gauge fixing, but also for defining an invariant measure on the space of $f(\tau)$ integrations.

With the above ingredients, the path integral is given by

$$
Z=\int \frac{\mathcal{D} f(\tau)^{\prime}}{f^{\prime}(\tau)} \exp \left[-S_{\text {hydro }}+\ldots\right]
$$

where $S_{\text {hydro }}$ is the effective action (5.17), describing the hydrodynamic modes (see the next section). The terms in the ellipsis denote subleading terms which get contribution from the Faddeev-Popov determinant mentioned above and discussed in detail in appendix F. The integration measure is the invariant integration measure in the space of $f(\tau)$ functions. The prime on the measure denotes the exclusion of the integration over $\mathbb{S L}(2, \mathbb{R})$ degrees of freedom due to the treatment of $\mathbb{S L}(2, \mathbb{R})$ modes discussed above. ${ }^{20}$

\section{Action of hydrodynamics modes}

We now compute the on-shell action of the above geometries to determine the contribution of the large diffeomorphisms discussed in this section to the partition function of the system.

\footnotetext{
${ }^{19}$ This is the standard prescription followed in open-string path integral computations. See also the relevant discussion in [5].

${ }^{20}$ This measure should appear from a proper treatment of the Faddeev-Popov procedure which is sketched in appendix F. We leave details of this to subsequent work.
} 


\subsection{Boundary action}

We know from our analysis of equations of motion in appendix $\mathrm{C}$ that all of $\mathrm{AAdS}_{2}$ geometries satisfy the bulk equations of motion. Thus we can safely anticipate that the major contribution to the action of hydrodynamics modes will come from the boundary terms in (3.12). The boundary terms of the action are given by,

$$
S_{L}^{\mathrm{bdy}}[\phi, \hat{g}]=-\frac{1}{4 \pi b^{2}}\left[2 \int_{\partial \Gamma} \sqrt{\hat{\gamma}} \hat{\mathcal{K}} \phi+\int_{\partial \Gamma} \sqrt{\hat{\gamma}} \hat{n}^{\mu} \phi \partial_{\mu} \phi\right]
$$

The second term above doesn't contribute at the leading order. The contribution of this term starts at, $\mathcal{O}(\delta g)^{2}$ and hence won't contribute to the leading order answers that we subsequently compute.

We also emphasize on the correct way to regulate the geometries for the subsequent computations. To keep our notations unambiguous, we will denote the coordinates of $\mathrm{AdS}_{2}$ by $\tilde{\zeta}, \tilde{\tau}$ and that of $\mathrm{AAdS}_{2}$ geometries by $\zeta, \tau$. We know that $\mathrm{AAdS}_{2}$ geometries are related to $\mathrm{AdS}_{2}$ geometry by large diffeomorphisms. If we put a radial cut-off in $\operatorname{AdS}_{2}$ at $\tilde{\zeta}=\delta$ and apply these large diffeomorphisms, then the cut-off at constant $\tilde{\zeta}$ is mapped to some wiggly-curves in $\zeta$ - $\tau$ coordinates, ${ }^{21}$

$$
\begin{aligned}
\delta & =\frac{4 \zeta f^{\prime}(\tau)^{3}}{4 f^{\prime}(\tau)^{2}+\zeta^{2} f^{\prime \prime}(\tau)^{2}} \\
\Rightarrow \zeta & =\frac{2 f^{\prime}(\tau)}{\delta f^{\prime \prime}(\tau)^{2}}\left[f^{\prime}(\tau)^{2}-\sqrt{f^{\prime}(\tau)^{4}-\delta^{2} f^{\prime \prime}(\tau)^{2}}\right]
\end{aligned}
$$

These are the same wiggles as discussed in [24]. To consider physically distinct geometries in $\zeta$ - $\tau$ coordinates, we put a cut-off at $\zeta=\delta$ and compare the action with that of geometry corresponding to $\tilde{\zeta}=\delta$.

In $\mathbf{A d S}_{\mathbf{2}}$. On the boundary $\tilde{\zeta}=\delta, \sqrt{\hat{\gamma}} \hat{\mathcal{K}}=\frac{1}{\delta}$

$$
\begin{aligned}
S_{L}^{\mathrm{bdy}}\left[\tilde{\phi}, \tilde{g}_{\alpha \beta}\right] & =-\frac{1}{2 \pi b^{2}} \int_{\partial \Gamma} \sqrt{\hat{\gamma}} \hat{\mathcal{K}} \tilde{\phi} \\
& =-\frac{1}{2 \pi b^{2}} \int d \tilde{\tau}\left[\left(\frac{\delta g(i \tilde{\tau})}{\delta^{2}}-\frac{1}{2} \delta g^{\prime \prime}(i \tilde{\tau})+\mathcal{O}\left(\delta^{2}\right)\right)+\mathcal{O}\left[\delta g(i \tilde{\tau})^{2}\right]\right]
\end{aligned}
$$

where, $\delta g(\tilde{\tau})$ was defined in (3.11). To be able to compare with the $\mathrm{AAdS}_{2}$ answer later, we do the coordinate transformation from $\tilde{\tau} \rightarrow \tau$ coordinates,

$$
\begin{aligned}
S_{L}^{\mathrm{bdy}}\left[\tilde{\phi}, \tilde{g}_{\alpha \beta}\right] & =-\frac{1}{2 \pi b^{2}} \int_{\partial \Gamma} \sqrt{\hat{\gamma}} \hat{\mathcal{K}} \tilde{\phi} \\
& =-\frac{1}{2 \pi b^{2} \delta} \int d \tau \frac{\partial \tilde{\tau}(\tau)}{\partial \tau}\left[\left(\frac{\delta g(i \tilde{\tau}(\tau))}{\delta}-\frac{\delta}{2} \delta g^{\prime \prime}(i \tilde{\tau}(\tau))+\mathcal{O}\left(\delta^{2}\right)\right)+\mathcal{O}\left[\delta g(i \tilde{\tau}(\tau))^{2}\right]\right] \\
\tilde{\tau} & =f(\tau)-\frac{f^{\prime}(\tau)^{2}}{f^{\prime \prime}(\tau)}\left(1-\sqrt{1-\delta^{2}\left(\frac{f^{\prime \prime}(\tau)}{f^{\prime}(\tau)^{2}}\right)^{2}}\right)
\end{aligned}
$$

\footnotetext{
${ }^{21}$ There are two solutions for $\zeta$ satisfying $\tilde{\zeta}=\delta$ (because the second equation in (4.5) is a quadratic in $\zeta$ ), one of which doesn't satisfy the boundary condition, $\zeta \rightarrow 0$ as $\delta \rightarrow 0$.
} 
Here, it is important to note that we need to implement the coordinate transformation at the $\tilde{\zeta}=\delta$ slice. To this effect, we need to solve for $\zeta$ at $\tilde{\zeta}=\delta$ using the second equation in (4.5) and substitute it back in the first equation there.

In $\mathbf{A A d S}_{2} \cdot \quad$ On the boundary $\zeta=\delta, \sqrt{\hat{\gamma}} \hat{\mathcal{K}}=\frac{1}{\delta}+\delta \frac{\{f(\tau), \tau\}}{2}$

$$
\begin{aligned}
S_{L}^{\text {bdy }}\left[\phi, g_{\alpha \beta}\right]= & -\frac{1}{2 \pi b^{2}} \int_{\partial \Gamma} \sqrt{\hat{\gamma}} \hat{\mathcal{K}} \phi \\
= & -\frac{1}{2 \pi b^{2}} \int d \tau\left[\frac{1}{\delta}+\delta \frac{\{f(\tau), \tau\}}{2}\right] \times\left[\left(\frac{1}{\delta} \frac{\delta g(i f(\tau))}{f^{\prime}(\tau)}\right.\right. \\
& -\delta \frac{\left(-\delta \mathrm{g}(i f(\tau)) f^{\prime \prime}(\tau)^{2}+2 f^{\prime}(\tau)^{4} \delta \mathrm{g}^{\prime \prime}(i f(\tau))+2 i f^{\prime}(\tau)^{2} f^{\prime \prime}(\tau) \delta \mathrm{g}^{\prime}(i f(\tau))\right)}{4 f^{\prime}(\tau)^{3}} \\
& \left.\left.+\mathcal{O}\left(\delta^{2}\right)\right)+\mathcal{O}\left[\delta g(i f(\tau))^{2}\right]\right]
\end{aligned}
$$

Hence,

$$
\begin{aligned}
\delta S_{L}^{\mathrm{bdy}}=S_{L}^{\mathrm{bdy}}\left[\phi, g_{\alpha \beta}\right] & -S_{L}^{\mathrm{bdy}}\left[\tilde{\phi}, \tilde{g}_{\alpha \beta}\right] \\
=\frac{1}{2 \pi b^{2}} \int d \tau[ & \left(\frac{\delta g(i f(\tau))}{\delta^{2}}\left(f^{\prime}(\tau)-\frac{1}{f^{\prime}(\tau)}\right)-\frac{\delta g(i f(\tau))}{f^{\prime}(\tau)}\{f(\tau), \tau\}\right. \\
& \left.\left.\quad+\mathcal{O}\left(\delta^{2}\right)\right)+\mathcal{O}\left[\delta g(i f(\tau))^{2}\right]\right]
\end{aligned}
$$

The $O\left(1 / \delta^{2}\right)$ divergent term can be subtracted by introducing following counterterm in the action (3.13),

$$
S_{\mathrm{ct}}=\frac{4 \sqrt{\pi \mu}}{4 \pi b^{2}} \int_{\partial \Gamma} \sqrt{\hat{\gamma}} \phi
$$

which essentially replaces $\sqrt{\hat{\gamma}} \hat{\mathcal{K}} \rightarrow \sqrt{\hat{\gamma}}(\hat{\mathcal{K}}-1) .^{22}$ To the linear order in $\delta g$ under consideration here, this is the same as the fully covariant counterterm $-4 \sqrt{\pi \mu} \frac{1}{4 \pi b^{2}} \int_{\partial \Gamma} \sqrt{\gamma} \frac{1}{\square} R$. The finite part of the answer is,

$$
\delta S_{L}^{\mathrm{bdy}}=-\frac{1}{2 \pi b^{2}} \int d \tau \frac{\delta g(i f(\tau))}{f^{\prime}(\tau)}\{f(\tau), \tau\}=\frac{1}{2 \pi b^{2}} \int d \tilde{\tau} \delta g(i \tilde{\tau})\{\tilde{f}(\tilde{\tau}), \tilde{\tau}\}
$$

Here the third term is written in terms of the $\tilde{\tau}$ coordinate, the boundary coordinate of the unperturbed $\mathrm{AdS}_{2} \cdot{ }^{23}$ Also, note that we have defined $\tilde{f}(\tilde{\tau})=\tau$ as the reparametrized coordinate starting with the unperturbed $\mathrm{AdS}_{2}$ coordinate $\tilde{\tau} .{ }^{24}$

\footnotetext{
${ }^{22} \mathrm{~A}$ similar counterterm is also implied in [5] in removing a quadratic divergence from their computation of the Schwarzian term.

${ }^{23}$ In going from the second expression to the third term, we have first transformed to the time coordinate $\tilde{\tau}=f(\tau)$, with $\tau=\tilde{f}(\tilde{\tau}), \tilde{f} \equiv f^{-1}$, and used the Schwarzian composition rule

$$
\{\tilde{f}(f(\tau)), \tau\}=\{\tilde{f}(f(\tau)), f(\tau)\} f^{\prime}(\tau)^{2}+\{f(\tau), \tau\}=\{\tilde{f}(\tilde{\tau}), \tilde{\tau}\} f^{\prime}(\tau)^{2}+\{f(\tau), \tau\},
$$

The l.h.s. equals $\{\tau, \tau\}$ and vanishes.

${ }^{24}$ It is important to note that the large diffeomorphism $\tilde{f}$ is what corresponds to the pseudo-NambuGoldstone mode $f$ of the SYK model.
} 
The function $\delta g(\tau)$ is given by (3.11). As indicated below that equation, we will henceforth choose $\delta g=$ constant. One might wonder if one can absorb the $\tau$ and $\tau^{2}$ deformations in $\delta g$, parameterized by $\delta a^{I}$ and $\delta c^{I}$, by a possible reparameterization of the boundary coordinate $\tau$; this, however, turns out impossible for any value of these parameters since the corresponding transformation turns out to be singular. Thus, the $\delta b^{I}, \delta a^{I}, \delta c^{I}$ represent different physics, and we will find that it is only the $\delta b^{I}$ deformation, that is, a constant $\delta g$, which will correspond to the SYK model. We will see that the nonnormalizable mode corresponding to constant $\delta g$, will correspond to the irrelevant coupling $1 / J$ of the SYK model. As remarked before, it is an important open question what the other parameters $\delta a^{I}$ and $\delta c^{I}$ correspond to.

In section 7 we will do the detailed matching with the boundary field theory. Note that the $\mathbb{S L}(2, \mathbb{R})$ transformations that correspond to the 'global conformal transformations' of one dimensional space remain the symmetry of this action. We have presented a discussion of the correct measure of integration over the $\tilde{f}(\tau)$ modes in subsection 4.1 and appendix $\mathrm{F}$.

\subsection{Bulk action}

The bulk part of the Liouville action is,

$$
\begin{aligned}
S_{L}^{\text {bulk }}[\phi, \hat{g}] & =-\frac{1}{4 \pi b^{2}} \int_{\Gamma} \sqrt{\hat{g}}\left(\hat{g}^{\alpha \beta} \partial_{\alpha} \phi \partial_{\beta} \phi+\hat{R} \phi+4 \pi \mu e^{2 \phi}\right) \\
& =-\frac{1}{4 \pi b^{2}} \int_{\partial \Gamma} \sqrt{\hat{\gamma}} \hat{n}^{\alpha} \phi \partial_{\alpha} \phi-\frac{1}{4 \pi b^{2}} \int_{\Gamma} \sqrt{\hat{g}}\left(-\phi \hat{\square} \phi+\hat{R} \phi+4 \pi \mu e^{2 \phi}\right) \\
& =-\frac{1}{4 \pi b^{2}} \int_{\partial \Gamma} \sqrt{\hat{\gamma}} \hat{n}^{\alpha} \phi \partial_{\alpha} \phi-\frac{1}{4 \pi b^{2}} \int_{\Gamma} \sqrt{\hat{g}}\left(\frac{1}{2} \hat{R} \phi+4 \pi \mu e^{2 \phi}(1-\phi)\right) \\
& =-\frac{1}{4 \pi b^{2}} \int_{\partial \Gamma} \sqrt{\hat{\gamma}} \hat{n}^{\alpha} \phi \partial_{\alpha} \phi-\frac{\mu}{b^{2}} \int_{\Gamma} \sqrt{\hat{g}}\left(-\phi+e^{2 \phi}(1-\phi)\right)
\end{aligned}
$$

here in the second line we have shifted the derivatives, while in the second term we have used the equation of motion of the $\phi$ field, $\hat{\square} \phi=\frac{1}{2} \hat{R}+4 \pi \mu e^{2 \phi}$. In the last line we have substituted the value of $\hat{R}=-8 \pi \mu$. The first term in the above equation contributes at subleading order, as argued under (5.1). Using the on-shell value of the $\phi$ we evaluate the above action in $\mathrm{AdS}_{2}$ and $\mathrm{AAdS}_{2}$ backgrounds.

In $\mathbf{A d S}_{\mathbf{2}}$. For $\mathrm{AdS}_{2}$ background metric, the action is given by,

$$
\begin{aligned}
S_{L}^{\mathrm{bulk}}\left[\tilde{\phi}, \tilde{g}_{\alpha \beta}\right] & =-\frac{\mu}{b^{2}} \int_{-\infty}^{\infty} d \tilde{\tau} \int_{\tilde{\zeta}=\delta}^{\infty} d \tilde{\zeta} \sqrt{\tilde{g}}\left(-\tilde{\phi}+e^{2 \tilde{\phi}}(1-\tilde{\phi})\right) \\
& =-\frac{\mu}{b^{2}} \int_{-\infty}^{\infty} d \tau \int_{\zeta>\partial \Gamma}^{\infty} d \zeta \sqrt{g}\left(-\phi+e^{2 \phi}(1-\phi)\right)
\end{aligned}
$$

Here, in the second line we have used the coordinate transformations, (4.5) and the boundary in $\zeta$ coordinates is now given by the wiggly curve, (5.2),

$$
\partial \Gamma \equiv \zeta=\frac{2}{\delta f^{\prime \prime}(\tau)^{2}}\left[f^{\prime}(\tau)^{3}-\sqrt{f^{\prime}(\tau)^{6}-\delta^{2} f^{\prime}(\tau)^{2} f^{\prime \prime}(\tau)^{2}}\right]
$$




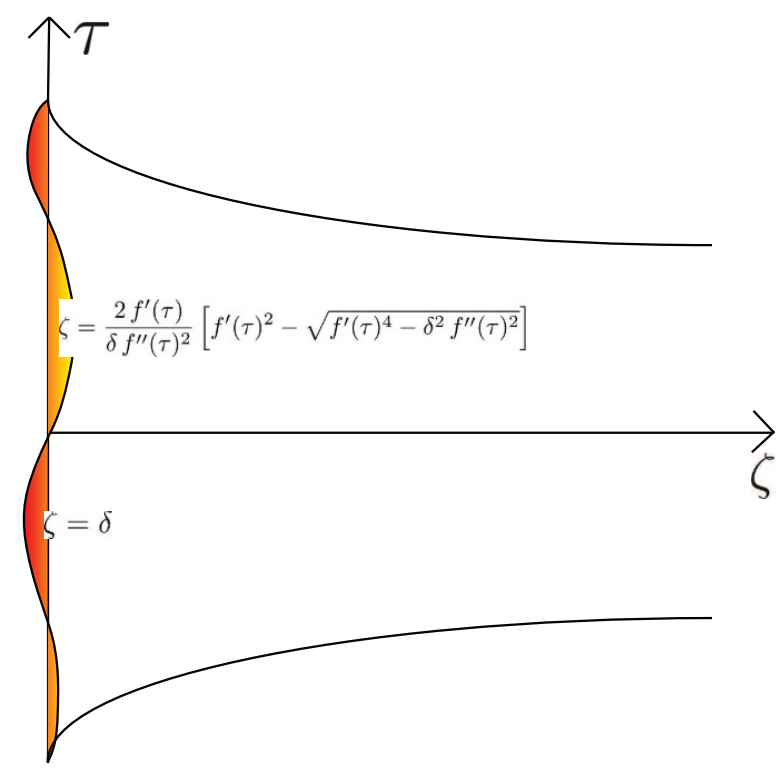

Figure 2. The difference in bulk action between $\mathrm{AdS}_{2}$ and $\mathrm{AAdS}_{2}$ geometries gets contribution only from the shaded region.

In $\mathbf{A A d S}_{2}$. Similarly for the $\mathrm{AAdS}_{2}$ background we have the action,

$$
S_{L}^{\mathrm{bulk}}\left[\phi, g_{\alpha \beta}\right]=-\frac{\mu}{b^{2}} \int_{-\infty}^{\infty} d \tau \int_{\zeta=\delta}^{\infty} d \zeta \sqrt{g}\left(-\phi+e^{2 \phi}(1-\phi)\right)
$$

Thus we have,

$$
\delta S_{L}^{\mathrm{bulk}}=S_{L}^{\mathrm{bulk}}\left[\phi, g_{\alpha \beta}\right]-S_{L}^{\mathrm{bulk}}\left[\tilde{\phi}, \tilde{g}_{\alpha \beta}\right]=-\frac{\mu}{b^{2}} \int_{-\infty}^{\infty} d \tau \int_{\delta}^{\zeta=\partial \Gamma} d \zeta \sqrt{g}\left(-\phi+e^{2 \phi}(1-\phi)\right)
$$

It is easy to approximate this expression close to the boundary of the geometry, i.e. when $\delta \rightarrow 0$. In that case the difference between $\zeta=\partial \Gamma$ and $\zeta=\delta$ reduces to a small strip as shown in figure 2. Moreover $\left(-\phi+e^{2 \phi}(1-\phi)\right) \sim 1+\mathcal{O}\left[\delta^{3}, \delta g(i \tau)^{2}\right]$ so that part of the integrand becomes trivial. Hence we can approximate the integrand by,

$$
\delta S_{L}^{\mathrm{bulk}}=\frac{1}{4 \pi b^{2}} \int_{-\infty}^{\infty} d \tilde{\tau}\left[\frac{1}{\delta}\left(\tilde{f}^{\prime}(\tilde{\tau})-1\right)+\frac{\delta}{4}\left(\frac{\left(2 \tilde{f}^{\prime}(\tilde{\tau})-3\right) \tilde{f}^{\prime \prime}(\tilde{\tau})^{2}-2 \tilde{f}^{\prime \prime \prime}(\tilde{\tau})\left(\tilde{f}^{\prime}(\tilde{\tau})-1\right) \tilde{f}^{\prime}(\tilde{\tau})}{\tilde{f}^{\prime}(\tau)^{3}}\right)\right]
$$

The first term is linearly divergent,${ }^{25}$ however while considering the coordinate transformations which approach identity transformations asymptotically this term integrates to zero. In other words if we consider a transformation, $\tilde{f}(\tilde{\tau})=\tilde{\tau}+\epsilon(\tilde{\tau}),{ }^{26}$ then $\tilde{f}^{\prime}(\tilde{\tau})=1+\epsilon^{\prime}(\tilde{\tau})$.

\footnotetext{
${ }^{25}$ We thank Shiraz Minwalla for a crucial discussion on this point.

${ }^{26}$ Here $\epsilon(\tilde{\tau})$ is not necessarily small, but just a rewriting of the coordinate transformations.
} 
In this case the first term becomes,

$$
\frac{1}{4 \pi b^{2}} \int_{-\infty}^{\infty} d \tilde{\tau} \frac{1}{\delta} \epsilon^{\prime}(\tilde{\tau})=\frac{1}{4 \pi b^{2} \delta}[\epsilon(\infty)-\epsilon(-\infty)]
$$

Clearly a good coordinate transformation has to be monotonically increasing. Additionally we require, for the transformation to remain invertible, that $\epsilon(\infty)=0=\epsilon(-\infty)$. In fact, the transformation that we use to map the theory on a line to a theory on a thermal circle is not of this kind and the regulation scheme adopted in that case is explained in section 6 . Leaving aside the issue of the regulation, the reparametrization of a thermal quantum mechanical theory can be achieved starting from a quantum mechanical theory in two steps: firstly, the straight line is mapped to a thermal circle using the map $\tilde{\tau}=\tan (\pi \tilde{\theta} / \beta)$; then, one reparametrizes the thermal circle with appropriate boundary conditions, ensuring reparametrization doesn't change the winding around the circle and is invertible. In this case, the Schwarzian action becomes [5, 24]:

$$
S_{\text {hydro }}^{\beta}=\frac{\delta g}{2 \pi b^{2}} \int d \tilde{\theta}\left\{\frac{\beta}{2} \tan \left(\pi \frac{f(\tilde{\theta})}{\beta}\right), \tilde{\theta}\right\}
$$

\subsection{Summary}

We thus find that the following low energy effective action (in the leading large $1 / b$ limit) for the 'hydrodynamic modes'

$$
S_{\text {hydro }}=\frac{\delta g}{2 \pi b^{2}} \int d \tilde{\tau}\{\tilde{f}(\tilde{\tau}), \tilde{\tau}\}
$$

In section 7 we will compare this with the Schwarzian term which appears in the SYK-type models.

It is important to mention that the bulk dual discussed in $[24,25]$, leads to a similar Schwarzian term starting from a dilaton gravity model, while the bulk dual discussed in this paper has only the metric field described by the Polyakov action. The source of the hydrodynamic modes in both cases involves the large diffeomorphisms which are nontrivial at the boundary. In a very recent paper [53], another proposal for a bulk dual has appeared which has a Liouville field and the Almheiri-Polchinski action [25]. They also appear to get a Schwarzian term rather differently, from the Liouville fluctuations similar to our functions $g(z), \bar{g}(\bar{z})$ in (3.6). However, as we found above, except for an $\mathbb{S L}(2, \mathbb{R})$ worth of degrees of freedom (see (3.8), (3.9)), these Liouville fluctuations are frozen by the Virasoro gauge conditions (3.7). It is also pertinent here to mention the theorems due to Schwarz and Pick [54]; these restrict the class of conformal transformations that map the boundary of Poincare half-plane to itself to only $\mathbb{S L}(2, \mathbb{R})$ transformations.

\section{Thermodynamic partition function from bulk dual}

In this section we compute the Euclidean bulk partition function in the classical limit for a black hole geometry. We use the standard prescription of [55] to renormalize the bulk 
partition function by subtracting the partition function of thermal $\mathrm{AdS}_{2}$ geometry from the Euclidean black-hole geometries that we describe below. ${ }^{27}$ Following [5, 24], we can do a reparametrization of the Euclidean time to study a field theory defined on a thermal circle of length $\beta$,

$$
\tilde{\tau}=\tan \left(\frac{\pi \tilde{\theta}}{\beta}\right)
$$

Using (4.6), we can compute the Euclidean geometry that is dual to the thermal field theory,

$$
d s^{2}=\frac{1}{4 \pi \mu \tilde{\zeta}^{2}}\left[d \tilde{\zeta}^{2}+\left(1-\pi^{2} \frac{\tilde{\zeta}^{2}}{\beta^{2}}\right)^{2} d \tilde{\tau}^{2}\right], \quad \tilde{\tau} \in\left(-\frac{\beta}{2}, \frac{\beta}{2}\right) \text { and } \tilde{\zeta} \in\left(0, \frac{\beta}{\pi}\right)
$$

This geometry is a capped $A d S_{2}$ geometry in two dimensions. There is no deficit angle near the horizon of the geometry, which can be easily checked by doing a near horizon expansion, $\tilde{\zeta}=\beta / \pi-\rho$,

$$
d s^{2} \sim \frac{\pi}{4 \mu \beta^{2}}\left[d \rho^{2}+4 \frac{\pi^{2}}{\beta^{2}} \rho^{2} d \tilde{\theta}^{2}\right]
$$

Analytically continuing this geometry to Lorentzian space we get,

$$
d s^{2}=\frac{1}{4 \pi \mu \tilde{\zeta}^{2}}\left[d \tilde{\zeta}^{2}-\left(1-\pi^{2} \frac{\tilde{\zeta}^{2}}{\beta^{2}}\right)^{2} d t^{2}\right]
$$

which is a geometry with a horizon at $\tilde{\zeta}=\beta / \pi$.

To get the free energy of the theory, we compute the on-shell bulk action for this geometry, but with a small non-normalizable deformation turned on (smallness is understood as explained in the previous section).

Bulk action. We first compute the bulk part of the action given in (3.13). The bulk part of the Liouville action is,

$$
\begin{aligned}
S_{L}^{\mathrm{bulk}}[\phi, \hat{g}] & =-\frac{1}{4 \pi b^{2}} \int_{\Gamma} \sqrt{\hat{g}}\left(\hat{g}^{\alpha \beta} \partial_{\alpha} \phi \partial_{\beta} \phi+\hat{R} \phi+4 \pi \mu e^{2 \phi}\right) \\
& =-\frac{1}{4 \pi b^{2}} \int_{\partial \Gamma} \sqrt{\hat{\gamma}} \hat{n}^{\alpha} \phi \partial_{\alpha} \phi-\frac{1}{4 \pi b^{2}} \int_{\Gamma} \sqrt{\hat{g}}\left(-\phi \hat{\square} \phi+\hat{R} \phi+4 \pi \mu e^{2 \phi}\right) \\
& =-\frac{1}{4 \pi b^{2}} \int_{\partial \Gamma} \sqrt{\hat{\gamma}} \hat{n}^{\alpha} \phi \partial_{\alpha} \phi-\frac{1}{4 \pi b^{2}} \int_{\Gamma} \sqrt{\hat{g}}\left(\frac{1}{2} \hat{R} \phi+4 \pi \mu e^{2 \phi}(1-\phi)\right) \\
& =-\frac{1}{4 \pi b^{2}} \int_{\partial \Gamma} \sqrt{\hat{\gamma}} \hat{n}^{\alpha} \phi \partial_{\alpha} \phi-\frac{\mu}{b^{2}} \int_{\Gamma} \sqrt{\hat{g}}\left(-\phi+e^{2 \phi}(1-\phi)\right)
\end{aligned}
$$

here in the second line we have shifted the derivatives, while in the third line we have used the equation of motion of $\phi$ field, $\hat{\square} \phi=\frac{1}{2} \hat{R}+4 \pi \mu e^{2 \phi}$. In the last line we have substituted the value of $\hat{R}=-8 \pi \mu$. The first boundary term in the last line combines with the

\footnotetext{
${ }^{27}$ Thermal $\mathrm{AdS}_{2}$ geometry is obtained simply by identifying the boundary time coordinate in (3.4) over a period $\beta$.
} 
boundary term already present in (3.13). However, these terms are not important for our analysis because they only contribute at $\mathcal{O}\left(\delta g^{3}\right)$. We don't have any leading contribution coming from the $\delta g$ modes from the bulk action,

$$
S_{L}^{\text {bulk }}\left[\tilde{\phi}, \tilde{g}_{\alpha \beta}\right]=\frac{1}{2 b^{2}}-\frac{\beta}{4 \pi b^{2} \delta}-\frac{\pi \delta}{4 b^{2} \beta}+\mathcal{O}\left(\delta g^{3}\right)
$$

As was the case with the previous Hydrodynamics calculation, all the divergent as well as finite terms above are cancelled by subtraction of the thermal $\mathrm{AdS}_{2}$ partition function. This is the standard prescription to regulate the partition function of the black hole geometries (see [55]). Thus the bulk contribution starts only at $\mathcal{O}\left(\delta g^{3}\right)$.

Boundary action. Computing the boundary terms of the action (3.13). Again, as argued above, the last term in (3.13) doesn't contribute at leading order. The term containing extrinsic curvature when evaluated on the boundary gives,

$$
S_{L}^{\mathrm{bdy}}\left[\tilde{\phi}, \tilde{g}_{\alpha \beta}\right]=\frac{\delta g}{2 b^{2} \beta}+\frac{\beta \delta g}{4 \pi^{2} b^{2} \delta^{2}}+\mathcal{O}\left(\delta g^{2}\right)
$$

In both the above expressions we have taken the boundary value of the $\delta g(i \tilde{\tau})$ field to be constant, as explained earlier, and have denoted it by $\delta g$. Again, the quadratically divergent term is cancelled by inclusion of the counterterm discussed in (5.8).

One last piece that needs to be evaluated is the bulk term $\int \sqrt{\hat{g}} \hat{R} \frac{1}{\hat{n}} \hat{R}$ that depends only on the background geometry. The Green's function in hyperbolic spaces is a well studied subject. In Green's function can be evaluated by taking a limit of the 'resolvent' of the Laplacian. ${ }^{28}$ The resolvent of the Laplacian on right half Poincare-plane, $\mathbb{H}$, is given by,

$$
\begin{aligned}
& \left(-\hat{\square}_{z}+4 \pi \mu s(s-1)\right) R_{\mathbb{H}}(s ; z, w)=4 \pi \mu \delta^{(2)}(z-w) \\
& R_{\mathbb{H}}(s ; z, w)=\frac{1}{4 \pi} \frac{\Gamma(s)^{2}}{\Gamma(2 s)}\left(1+\frac{|z-w|^{2}}{4 \operatorname{Re}(z) \operatorname{Re}(w)}\right)^{-s}{ }_{2} F_{1}\left[s, s ; 2 s ; \frac{1}{1+\frac{|z-w|^{2}}{4 \operatorname{Re}(z) \operatorname{Re}(w)}}\right]
\end{aligned}
$$

Here, $z, w$ are the complexified coordinates, $z=\zeta_{1}+i \tau_{1}$ and $w=\zeta_{2}+i \tau_{2}$. The $s \rightarrow 1$ limit of this function is,

$$
G\left(\left\{\zeta_{1}, \tau_{1}\right\} ;\left\{\zeta_{2}, \tau_{2}\right\}\right)=-\frac{1}{4 \pi} \log \left(1-\frac{4 \zeta_{1} \zeta_{2}}{\left(\zeta_{1}+\zeta_{2}\right)^{2}+\left(\tau_{1}-\tau_{2}\right)^{2}}\right)
$$

However, the above results are in $\mathbb{H}$, while we are interested in solving the Green's function for the geometry in (6.2). The Green's function can be obtained easily using the coordinate transformations in (4.5) with the choice of function in (6.1). We get,

$$
G=-\frac{1}{4 \pi} \log \left[1-\frac{8 \pi^{2} \beta^{2} \zeta_{1} \zeta_{2}}{\beta^{4}+\pi^{2} \beta^{2}\left(\zeta_{1}^{2}+4 \zeta_{1} \zeta_{2}+\zeta_{2}^{2}\right)-\left(\beta^{2}-\pi^{2} \zeta_{1}^{2}\right)\left(\beta^{2}-\pi^{2} \zeta_{2}^{2}\right) \cos \left(\frac{2 \pi\left(\theta_{1}-\theta_{2}\right)}{\beta}\right)+\pi^{4} \zeta_{1}^{2} \zeta_{2}^{2}}\right]
$$

\footnotetext{
${ }^{28} \mathrm{~A}$ resolvent in defined as the classical Green's function of the operator $-\square+4 \pi \mu s(s-1)$. Thus the required Green's function is the $s \rightarrow 1$ limit of the resolvent.
} 
With this Green's function we solve the

$$
\int \sqrt{\hat{g}} \hat{R} \frac{1}{\hat{\square}} \hat{R}=\int \sqrt{\hat{g}\left(\zeta_{1}, \tau_{1}\right)} \int \sqrt{\hat{g}\left(\zeta_{2}, \tau_{2}\right)} R_{1} G\left(\left\{\zeta_{1}, \tau_{1}\right\} ;\left\{\zeta_{2}, \tau_{2}\right\}\right) R_{2}
$$

term for the geometry, (6.2). We get,

$$
\int \sqrt{\hat{g}} \hat{R} \frac{1}{\hat{\square}} \hat{R}=\frac{\beta}{\pi b^{2} \delta}-\frac{2}{b^{2}} \log (\beta / \delta)+\frac{2 \log (4 \pi)-3}{b^{2}}-\frac{\pi \delta}{3 b^{2} \beta}
$$

Again, the linearly divergent piece that appears above is cancelled by the contribution coming from the thermal $\mathrm{AdS}_{2}$ partition function. ${ }^{29}$

Thus, the total action is

$$
\log (Z)=-\beta F=-\frac{2}{b^{2}} \log (\beta / \delta)+\frac{2 \log (4 \pi)-3}{b^{2}}+\frac{\delta g}{2 b^{2} \beta}+\mathcal{O}\left(\delta g^{2}\right)
$$

\section{Comparison with field theory}

In this section, we will make some remarks comparing our gravity dual we discussed above and the SYK model.

\subsection{Hydrodynamics and a double scaling}

The gravity dual leads to the following low energy effective action

$$
S_{\text {hydro }}=\frac{\delta g}{2 \pi b^{2}} \int d \tilde{\tau}\{\tilde{f}(\tilde{\tau}), \tilde{\tau}\}
$$

while the SYK model has the following expression for the same quantity $[1,5]$

$$
S_{\text {hydro }}=N \frac{\alpha(q)}{\mathcal{J}} \int d \tau\{\tilde{f}(\tau), \tau\}
$$

As we argued above, $\delta g$ plays the role of the explicit symmetry breaking parameter $1 / J$ in the SYK model. Further, the classical limit in the bulk model corresponds to $b \rightarrow 0$, which, therefore corresponds to the limit $N \rightarrow \infty$. Therefore, we identify these quantities up to constants, thus:

$$
\frac{1}{b^{2}}=c_{1} N, \quad \delta g=c_{2} \frac{1}{\mathcal{J}}
$$

For the two hydrodynamic expressions above to match, we need to have $c_{1} c_{2}=\alpha(q)$. A $q$-dependence in the coefficients $c_{1}, c_{2}$ may appear strange; however, it may indicate the existence of a double scaling in the theory. Note that at large $q, \alpha(q) a_{0} / q^{2}$ ( $a_{0}=$ constant). A possible choice of the coefficients is $c_{1}=\alpha(q), c_{2}=1$. In this case, we are essentially identifying

$$
\frac{1}{b^{2}}=a_{0} N / q^{2}, \quad \delta g=c_{2} \frac{1}{\mathcal{J}}
$$

Thus, if we take the limit $N \rightarrow \infty$, and $q^{2} / N$ fixed (cf. [18] appendix B), the corresponding scaled quantity corresponds to the bulk Newton's constant:

$$
q^{2} / N=a_{0} b^{2}
$$

\footnotetext{
${ }^{29}$ It can be seen easily by doing a similar computation using the Green's function, (B.12), on the thermal $\mathrm{AdS}_{2}$ geometry as discussed in appendix B.
} 


\subsection{Thermodynamics}

At low temperatures, the bulk partition function is given by (6.11), with a divergence of the form $\log (\beta / \delta)$. With the logarithmically divergent term we might typically be left with finite parts, say $P_{0}$, after cancellation of the divergence. The low temperature partition function will then be given by, ignoring subleading order terms in $\delta g / \delta$,

$$
\log (Z)=-\beta F=\frac{1}{b^{2}}\left[\left(-2 P_{0}+\frac{4 \log (4 \pi)-5}{2}\right)+\frac{\delta g}{2 \beta}+\mathcal{O}\left(\delta g^{2}\right)\right]
$$

The corresponding expression in the SYK model is $[5,18,19]$

$$
\log (Z)=-\beta F=N\left[\beta \mathcal{J} \frac{1}{q^{2}}+\frac{1}{2} \log 2-\frac{\pi^{2}}{4 q^{2}}+\frac{1}{\beta \mathcal{J}} \frac{\pi^{2}}{2 q^{2}}+O\left(\frac{1}{q^{4}}\right)\right]
$$

It is then possible that by suitably adjusting the finite part $P_{0}$ and the constant $c_{2}$ introduced above, one can match the zero-temperature entropy and the low temperature specific heat. The SYK zero-temperature entropy here does not seem to be universal; however, in the double scaling limit mentioned above, the $N / q^{2}$ term $i s$ universal.

A more detailed understanding of the low energy thermodynamics is clearly desirable and is under investigation presently.

\section{Discussion and open questions}

In this work, we arrive at a proposal for a gravity dual of the low energy sector of SYK-type models from symmetry considerations, more precisely from the fact that the coadjoint orbit action of the Diff group is the Polyakov action (2.7). We solve the classical equations of motion and find that the solutions are parametrized by a large diffeomorphism together with a specific conformal factor (value of the Liouville mode) representing a non-normalizable deformation. We compute the on-shell action which evaluates the classical contribution to $\log Z$. The computation leads to a Schwarzian action for the low energy hydrodynamic modes and a specific heat which is linear at low temperatures. Thus, the low energy behaviour of our proposed gravity dual reproduces that of SYK-type models.

We will end with some remarks about possible UV properties of the bulk dual. Recall that in usual AdS/CFT, such as in the example of $\mathcal{N}=4$ SYM theory on $S^{3} \times R$, states with spin $>2$ acquire very large anomalous dimensions $\gamma \sim\left(g_{\mathrm{YM}}^{2} N\right)^{1 / 4}$ at strong coupling ${ }^{30}$ The energy grows as $E \sim \gamma / R_{\text {AdS }}$ and the corresponding bulk state is identified as a string state with mass $m_{s}=\left(g_{\mathrm{YM}}^{2} N\right)^{1 / 4} / R_{\mathrm{AdS}}$. This corresponds to the fact that the UV completion of the gravity theory is string theory in AdS. In case of SYK-type models, the anomalous dimensions of operators with spin higher than two, which form an approximately Regge trajectory, remain $O(1)$ even at strong coupling. From the point of the bulk dual, the usual mass-dimension formula (which follows by using the relation between the $\mathrm{AdS}_{2}$ Laplacian and Casimir of $\mathbb{S L}(2, \mathbb{R})$ ) implies $E \sim \Delta / R_{\mathrm{AdS}}$ (in our model, $R_{\mathrm{AdS}} \sim 1 / \sqrt{\mu}$, see (3.2)). If we wish to identify the 'Reggeons' with possible string states, this would imply that the 'string length' is of the same order as the AdS radius. It is not clear what such a dual string theory of light strings is. On the other hand, the spectrum of these massive modes

\footnotetext{
${ }^{30}$ Primary operators with spin $\leq 2$ retain $O(1)$ anomalous dimensions. These correspond to spherical harmonics of gravitons with $E \sim O(1) / R_{\text {AdS }}$.
} 
suggests that it may be possible to incorporate these states in our bulk dual by adding to the Polyakov action (2.7) an infinite number of matter fields $\eta_{r}$ minimally coupled to the metric (see [24] for related ideas), with masses $m_{r}$ given in terms of the conformal dimensions $\Delta_{r}$. In such a scenario, the Polyakov action (2.7) would still continue to represent the physics of the 'Nambu-Goldstone' modes. The full action will have the structure

$$
S=S_{\text {cov }}[g]+S_{\text {matter }}\left[g,\left\{\eta_{r}\right\}\right]
$$

where $S_{\text {cov }}[g]$ is the Polyakov action, given by (2.7). The matter action

$$
\begin{aligned}
S_{\text {matter }}\left[g,\left\{\eta_{r}\right\}\right] & =\frac{1}{2} \int_{\Gamma} \sqrt{g}\left[\sum_{r}\left(g^{\alpha \beta} \partial_{\alpha} \eta_{r} \partial_{\beta} \eta_{r}+m_{r}^{2} \eta_{r}^{2}\right)+\ldots\right] \\
& =\frac{1}{2} \int_{\Gamma} \sqrt{\hat{g}}\left[\sum_{r}\left(\hat{g}^{\alpha \beta} \partial_{\alpha} \eta_{r} \partial_{\beta} \eta_{r}+m_{r}^{2} e^{2 \phi} \eta_{r}^{2}\right)+\ldots\right]
\end{aligned}
$$

where in the second step, we have used (4.7). Note that since the metric $\hat{g}$ contains the Nambu-Goldstone modes $f$ (see (4.6)), the above action automatically incorporates a coupling between these modes and the higher mass modes $\eta_{r}$; this fact plays an important role in computing the chaotic growth of the out-of-time correlator. Using the action (8.1) we can derive the exponentially growing behaviour of the out-of-time ordered 4-point functions, $\langle\mathcal{O}(\tau) \mathcal{O}(0) \mathcal{O}(\tau) \mathcal{O}(0)\rangle$, which gives the Lyapunov exponent, $2 \pi / \beta$, consistent with the bound on chaos derived in [26]. Note also the appearance of the Liouville factor in the mass term (this is to be contrasted with proposed bulk duals based on Jackiw-Teitelboim models, e.g. in [24]). This implies subleading correction to the mass term proportional to $1 / J$ (see $(4.7))$. However, as shown in $[5,56]$ one doesn't need to break the conformal symmetry explicitly to study the physics of these excited states. In fact, the $1 / J$ corrections for these states are truly subleading. The terms in the ellipsis above denote interaction terms, which are suppressed in large $N$ counting. Whether the procedure of incorporating bulk fields outlined above can be consistently extended to an interacting level with local interactions in the bulk, of course, remains an open question.

\section{Acknowledgments}

We would like to thank L. Alvarez-Gaume, T. Batista, S. Bhattacharya, A. Dabholkar, A. Dhar, R. Gopakumar, D. Gross, D. Harlow, S. Jain, J. Maldacena, S. Minwalla, R. Poojary, S. Sachdev, A. Sen, A. Sinha, R. Soni, J. Sonner, D. Stanford, A. Strominger, S. Trivedi, H. Verlinde and E. Witten. G.M. would like to thank participants of the meetings String Theory: Past and Present, ICTS-TIFR, Bangalore, 11-13 January 2017 [57], for stimulating discussions where part of this work was presented. The work of G.M and P.N. was supported in part by Infosys Endowment for the study of the Quantum Structure of Space Time. S.R.W. would like to thank Theory Division of CERN Geneva where part of this work was done. S.R.W. would like to acknowledge the support of the Infosys Foundation Homi Bhabha Chair at ICTS. We thank S. Minwalla for insightful comments on the original draft of this paper.

We would like to thank D. Stanford and E. Witten for illuminating correspondences on the first version. 


\section{A Some identities}

For $g_{\alpha \beta}=e^{2 \phi} \hat{g}_{\alpha \beta}$

$$
\begin{aligned}
R & =e^{-2 \phi}(\hat{R}-2 \hat{\square} \phi) \\
\square & =e^{-2 \phi} \hat{\square} \\
g:=\operatorname{det}\left(g_{\alpha \beta}\right) & =e^{4 \phi} \hat{g} \\
n_{\mu} & =e^{\phi} \hat{n}_{\mu} \\
n^{\mu} & =e^{-\phi} n^{\mu} \\
\gamma:=\operatorname{det}\left\{\gamma_{\alpha \beta}\right\} & =e^{2 \phi} \hat{\gamma} \\
\sqrt{\gamma} \mathcal{K} & =\sqrt{\hat{\gamma}}\left(\hat{\mathcal{K}}+\hat{n}^{\mu} \partial_{\mu} \phi\right)
\end{aligned}
$$

\section{B Green's function of Laplacian in $\mathrm{AdS}_{2}$}

Green's functions in hyperbolic spaces are well studied. Therefore, in this appendix, following [58], we only provide a quick review of some results that are important for this paper. In the Poincare half plane, $\mathbb{H}$, the Laplacian is given by,

$$
\hat{\square}=\zeta^{2}\left(\partial_{\zeta}^{2}+\partial_{\tau}^{2}\right)
$$

We are interested in solving the Green's function equation,

$$
\hat{\square} G\left(\vec{x}, \vec{x}^{\prime}\right)=\zeta^{2} \delta^{(2)}\left(\vec{x}-\vec{x}^{\prime}\right)
$$

It is convenient to work with the coordinates, $z=\zeta+i \tau, \bar{z}=\zeta-i \tau$. Geodesic distances between two points, $z, z^{\prime}$, on $\mathbb{H}$ are given by,

$$
d\left(z, z^{\prime}\right)=\frac{1}{\sqrt{4 \pi \mu}} \arccos \left(1+\frac{\left|z-z^{\prime}\right|^{2}}{2 \operatorname{Re}[z] \operatorname{Re}\left[z^{\prime}\right]}\right)
$$

Hyperbolic symmetry implies that the Green's function depends only on the geodesic distance, $G\left(z, z^{\prime}\right)=f(d)$. Switching to geodesic polar coordinates centered around $z^{\prime}$,

$$
d s^{2}=d r^{2}+\sinh ^{2}(2 \sqrt{\pi \mu} r) d \theta^{2}
$$

In these coordinates, (B.2) becomes,

$$
\left[\frac{1}{\sinh (2 \sqrt{\pi \mu} r)} \partial_{r}\left(\sinh (2 \sqrt{\pi \mu} r) \partial_{r}\right)\right] f(r)=\frac{\delta(r)}{\sinh (2 \sqrt{\pi \mu} r)}
$$

We regulate the above equation by first solving the resolvent for the operator $-\square+4 \pi \mu s(s-$ 1 ), and then taking the limit, $s \rightarrow 1$. Moreover, we first solve the homogeneous condition and then impose an appropriate condition on the discontinuity of the resolvent at origin to solve for the Green's function. The solution to the regulated homogeneous equation,

$$
\left[\frac{1}{\sinh (2 \sqrt{\pi \mu} r)} \partial_{r}\left(\sinh (2 \sqrt{\pi \mu} r) \partial_{r}\right)+s(s-1)\right] f_{s}(r)=0
$$


is given by,

$$
f_{s}(r)=a_{1} Q_{s-1}(\cosh (2 \sqrt{\pi \mu} r))+a_{2} P_{s-1}(\cosh (2 \sqrt{\pi \mu} r))
$$

where, $P_{s}, Q_{s}$ are Legendre functions of first and second kind respectively, and $a_{1}, a_{2}$ are some constant of integrations. To fix the normalization and the discontinuity at the origin, we substitute (B.7) into (B.5) and integrate on both sides. This fixes the solution for the resolvent to be,

$$
\begin{aligned}
f_{s}(r) & =-\frac{1}{2 \pi} Q_{s-1}(\cosh (2 \sqrt{\pi \mu} r)) \\
G_{s}\left(z, z^{\prime}\right) & =-\frac{1}{2 \pi} Q_{s-1}\left(1+\frac{\left|z-z^{\prime}\right|^{2}}{2 \operatorname{Re}[z] \operatorname{Re}\left[z^{\prime}\right]}\right) \\
& =-\frac{\Gamma(s)^{2}}{4 \pi \Gamma(2 s)}\left(1+\frac{\left|z-z^{\prime}\right|^{2}}{4 \operatorname{Re}[z] \operatorname{Re}\left[z^{\prime}\right]}\right)^{-s}{ }_{2} F_{1}\left(s, s ; 2 s ;\left(1+\frac{\left|z-z^{\prime}\right|^{2}}{4 \operatorname{Re}[z] \operatorname{Re}\left[z^{\prime}\right]}\right)^{-1}\right)
\end{aligned}
$$

Taking $s \rightarrow 1$, the Green's function is given by,

$$
G\left(z, z^{\prime}\right)=\frac{1}{4 \pi} \log \left[1-\left(1+\frac{\left|z-z^{\prime}\right|^{2}}{4 \operatorname{Re}[z] \operatorname{Re}\left[z^{\prime}\right]}\right)^{-1}\right]
$$

In terms of the $\zeta-\tau$ coordinates, this is,

$$
G\left(\left\{\zeta_{1}, \tau_{1}\right\},\left\{\zeta_{2}, \tau_{2}\right\}\right)=\frac{1}{4 \pi} \log \left[\frac{\left(\zeta_{1}-\zeta_{2}\right)^{2}+\left(\tau_{1}-\tau_{2}\right)^{2}}{\left(\zeta_{1}+\zeta_{2}\right)^{2}+\left(\tau_{1}-\tau_{2}\right)^{2}}\right]
$$

The Green's function is quite instructive in this form. It is same as the flat space Green's function in 2-dimensions with an additional contribution coming from the 'mirror charge' at $\left\{-\zeta_{2}, \tau_{2}\right\}$. This is not surprising because $\mathrm{AdS}_{2}$ is Weyl scaled flat metric and hence has the same Green's function up to imposition of boundary conditions.

\section{B.1 Green's function for thermal $\mathrm{AdS}_{2}$}

Thermal $\mathrm{AdS}_{2}$ is defined by periodic identification of $\tau$ coordinate over a length $\beta$. Thus the metric remains same as pure $\mathrm{AdS}_{2}$ and so does the Laplacian given in (B.1). However, now the Green's function should be invariant under the shift $\Delta \tau=\tau_{1}-\tau_{2} \rightarrow \Delta \tau+n \beta$, with $n \in \mathbb{Z}$. This can be achieved by taking using the method of images,

$$
G_{\text {thermal }}\left(\left\{\zeta_{1}, \tau_{1}\right\},\left\{\zeta_{2}, \tau_{2}\right\}\right)=\frac{1}{4 \pi} \sum_{n=-\infty}^{\infty} \log \left[\frac{\left(\zeta_{1}-\zeta_{2}\right)^{2}+(\Delta \tau+n \beta)^{2}}{\left(\zeta_{1}+\zeta_{2}\right)^{2}+(\Delta \tau+n \beta)^{2}}\right]
$$

This sum can be computed explicitly,

$$
G_{\text {thermal }}\left(\left\{\zeta_{1}, \tau_{1}\right\},\left\{\zeta_{2}, \tau_{2}\right\}\right)=\frac{1}{4 \pi} \log \left[\frac{\cosh \left(\frac{2 \pi\left(\zeta_{1}-\zeta_{2}\right)}{\beta}\right)-\cos \left(\frac{2 \pi \Delta \tau}{\beta}\right)}{\cosh \left(\frac{2 \pi\left(\zeta_{1}+\zeta_{2}\right)}{\beta}\right)-\cos \left(\frac{2 \pi \Delta \tau}{\beta}\right)}\right]
$$

This Green's function was used in the computations of the partition function in section 6 which was then subtracted from the partition function in black hole geometries discussed in that section. 


\section{Variation of the induced gravity (Polyakov) action}

In this appendix we will study the exact variation of the Polyakov action, (2.7). We haven't found a discussion of these covariant equations of motion anywhere in literature, we think that it might have been worked out personally, they haven't been presented in published form. Since the action is non-local, so are the equations of motion. ${ }^{31}$ While we won't be solving the equations in full generality, we show,

1. That the diagonal part of the equations of motion are the same as the one we obtain for the Liouville mode, $\phi$, in conformal gauge. These is the equation of motion that one obtains for Liouville field theory with a background metric, $\hat{g}$.

2. $\mathrm{AdS}_{2}$ and $\mathrm{AAdS}_{2}$ geometries that we have discussed in the paper satisfy the equations of motion.

3. The most general solutions $([45,46])$ of the Liouville mode, $\phi$, in $\mathrm{AdS}_{2}$ background,

$$
\phi=\frac{1}{2} \log \left[(z+\bar{z})^{2} \frac{\partial g(z) \bar{\partial} \bar{g}(\bar{z})}{(g(z)+\bar{g}(\bar{z}))^{2}}\right]
$$

obtain further constraints from the equations of motion. That is not surprising because the above solutions were obtained from solving only the Liouville equation. This also bodes well with the degree of freedom counting in $2 \mathrm{~d}$ theory of gravity. These constraints force the solutions of $g(z), \bar{g}(\bar{z})$ to be $\mathbb{S L}(2, \mathbb{C})$ transformations of complex plane, (3.8). However, the boundary conditions reduce it to $\mathbb{S L}(2, \mathbb{R})$ transformations, which are the isometries of the geometries that we are interested in. The remaining solutions that don't satisfy the boundary conditions are what we call non-normalizable solutions.

4. This exercise also justifies the boundary terms that we have introduced in (2.7) that we have argued are required for a well defined variational principle.

We use following notations to avoid clutter in the forthcoming equations:

$$
\begin{aligned}
& \int_{\Gamma}^{x} \equiv \int_{\Gamma} d^{2} x \sqrt{g(x)} \\
& \int_{\partial \Gamma}^{s} \equiv \int_{\partial \Gamma} d s \sqrt{\gamma(s)} \text { where } s \text { is the boundary coordinate } \\
& G(x, y) \text { is the Green's function of the Laplacian satisfying, } \square^{(x)} G(x, y)=\frac{\delta^{2}(x-y)}{\sqrt{g(x)}}
\end{aligned}
$$

$\nabla_{\mu}^{(x)}$ denotes the covariant derivative with respect to variable $x$

\footnotetext{
${ }^{31}$ This also makes this section pretty ugly in terms of the equations.
} 
Bulk term. We start with varying the bulk term in (2.7).

$$
\begin{aligned}
\delta S_{\text {cov }}^{\text {bulk }}[g] & \frac{1}{16 \pi b^{2}} \int_{\Gamma} \delta\left(\sqrt{g}\left[R \frac{1}{\square} R-16 \pi \mu\right]\right) \\
= & \frac{1}{16 \pi b^{2}} \int_{\Gamma} d^{2} x \int_{\Gamma} d^{2} y \delta[\sqrt{g(x)} \sqrt{g(y)} R(x) G(x, y) R(y)]+\frac{1}{16 \pi b^{2}} \int_{\Gamma} d^{2} x \delta[\sqrt{g(x)}](-16 \pi \mu) \\
= & \frac{1}{16 \pi b^{2}} \int_{\Gamma} d^{2} x \int_{\Gamma} d^{2} y(2 \delta[\sqrt{g(x)} R(x)] \sqrt{g(y)} R(y) G(x, y)+\sqrt{g(x)} R(x) \sqrt{g(y)} R(y) \delta[G(x, y)]) \\
& +\frac{1}{16 \pi b^{2}} \int_{\Gamma} d^{2} x \delta[\sqrt{g(x)}](-16 \pi \mu)
\end{aligned}
$$

Here, in the last equation on r.h.s. we have used the symmetry of Green's function in $x-y$ coordinates to multiply the first term by 2 . In the above equation, the first and last term are very easy to compute while the second term is slightly more non-trivial. The variations of the Ricci scalar and metric determinant are:

$$
\begin{aligned}
\delta[\sqrt{g(x)}] & =-\frac{1}{2} \sqrt{g(x)} g_{\mu \nu}(x) \delta g^{\mu \nu}(x) \\
\delta[R(x)] & =R_{\mu \nu} \delta g^{\mu \nu}+\nabla_{\mu} v^{\mu} \\
\text { where, } v^{\sigma} & =g_{\mu \nu} \nabla^{\sigma}\left(\delta g^{\mu \nu}\right)-\nabla_{\alpha}\left(\delta g^{\alpha \sigma}\right)
\end{aligned}
$$

Henceforth, we are dropping the overall factor of $1 / 16 \pi b^{2}$ and will reinstate it at the end.

$$
\begin{aligned}
\delta S_{\mathrm{cov}}^{\mathrm{bulk}}[g]= & 2 \int_{\Gamma}^{x} \int_{\Gamma}^{y}\left(R_{\mu \nu}(x)-\frac{1}{2} g_{\mu \nu}(x) R(x)\right) \delta g^{\mu \nu}(x) R(y) G(x, y)+2 \int_{\Gamma}^{x} \int_{\Gamma}^{y} \nabla_{\mu}^{(x)} v^{\mu} R(y) G(x, y) \\
& +\int_{\Gamma}^{x} \int_{\Gamma}^{y} R(x) R(y) \delta[G(x, y)]+\int_{\Gamma}^{x} 8 \pi \mu g_{\mu \nu}(x) \delta g^{\mu \nu}(x) \\
= & 2 \int_{\Gamma}^{x} \int_{\Gamma}^{y} \nabla_{\sigma}^{(x)}\left[g_{\mu \nu}(x) \nabla_{(x)}^{\sigma}\left(\delta g^{\mu \nu}(x)\right)-\nabla_{\alpha}^{(x)}\left(\delta g^{\alpha \sigma}(x)\right)\right] R(y) G(x, y) \\
& +\int_{\Gamma}^{x} \int_{\Gamma}^{y} R(x) R(y) \delta[G(x, y)]+\int_{\Gamma}^{x} 8 \pi \mu g_{\mu \nu}(x) \delta g^{\mu \nu}(x)
\end{aligned}
$$

where, in the second line we have dropped the term containing Einstein tensor which is identically zero in 2 dimensions. Subsequently, we integrate by parts, keeping track of all the boundary terms that we pick in the process.

$$
\begin{aligned}
\delta S_{\mathrm{cov}}^{\mathrm{bulk}}[g]= & -2 \int_{\Gamma}^{x} \int_{\Gamma}^{y}\left[\nabla_{(x)}^{\sigma}\left(g_{\mu \nu}(x) \delta g^{\mu \nu}(x)\right)-\nabla_{\alpha}^{(x)}\left(\delta g^{\alpha \sigma}(x)\right)\right] R(y) \nabla_{\sigma}^{(x)} G(x, y) \\
& +2 \int_{\Gamma}^{y} \int_{\Gamma}^{x} \nabla_{\sigma}^{(x)}\left[v^{\sigma}(x) R(y) G(x, y)\right]+\int_{\Gamma}^{x} \int_{\Gamma}^{y} R(x) R(y) \delta[G(x, y)]+\int_{\Gamma}^{x} 8 \pi \mu g_{\mu \nu}(x) \delta g^{\mu \nu}(x) \\
= & -2 \int_{\Gamma}^{x} \int_{\Gamma}^{y}\left[\nabla_{(x)}^{\sigma}\left(g_{\mu \nu}(x) \delta g^{\mu \nu}(x)\right)-\nabla_{\alpha}^{(x)}\left(\delta g^{\alpha \sigma}(x)\right)\right] R(y) \nabla_{\sigma}^{(x)} G(x, y) \\
& +2 \int_{\Gamma}^{y} \int_{\partial \Gamma}^{s} \hat{n}_{\sigma}(s) v^{\sigma}(s) R(y) G(x, y)+\int_{\Gamma}^{x} \int_{\Gamma}^{y} R(x) R(y) \delta[G(x, y)]+\int_{\Gamma}^{x} 8 \pi \mu g_{\mu \nu}(x) \delta g^{\mu \nu}(x) \\
= & -4 \int_{\Gamma}^{y} \int_{\partial \Gamma}^{s} \delta \mathcal{K} R(y) G(x, y)-2 \int_{\Gamma}^{x} \int_{\Gamma}^{y} \nabla_{(x)}^{\sigma}\left(g_{\mu \nu}(x) \delta g^{\mu \nu}(x) R(y) \nabla_{\sigma}^{(x)} G(x, y)\right) \\
& +\int_{\Gamma}^{x} g_{\mu \nu}(x) \delta g^{\mu \nu}(x)(2 R(x)+8 \pi \mu)+2 \int_{\Gamma}^{x} \int_{\Gamma}^{y} \nabla_{\alpha}^{(x)}\left(\delta g^{\alpha \sigma}(x) R(y) \nabla_{\sigma}^{(x)} G(x, y)\right) \\
& -2 \int_{\Gamma}^{x} \int_{\Gamma}^{y} \delta g^{\alpha \sigma}(x) \nabla_{\alpha}^{(x)} \nabla_{\sigma}^{(x)} G(x, y) R(y)+\int_{\Gamma}^{x} \int_{\Gamma}^{y} R(x) R(y) \delta[G(x, y)]
\end{aligned}
$$


in the second line on r.h.s., we have used the Gauss's law to make the bulk integral into a surface integral. The first term in the second line is also the term that needs to be cancelled because it involves derivatives of variation of metric. Using $\hat{n}^{\sigma} v_{\sigma}=-2 \delta \mathcal{K}$, one can clearly see that this term is cancelled by the variation of second term in (2.7). In the third line, we have used integration by parts in the second term of the second line. We have obtained two boundary terms in the process ( $2^{\text {nd }}$ and $4^{\text {th }}$ term in the third line), both of which involve variation of the metric on the boundary, and under Dirichlet boundary condition, are zero. They will be dropped from here onwards.

$$
\begin{aligned}
\delta S_{\mathrm{cov}}^{\mathrm{bulk}}[g]= & -4 \int_{\Gamma}^{y} \int_{\partial \Gamma}^{s} \delta \mathcal{K} R(y) G(x, y)+\int_{\Gamma}^{x} g_{\mu \nu}(x) \delta g^{\mu \nu}(x)(2 R(x)+8 \pi \mu) \\
& -2 \int_{\Gamma}^{x} \int_{\Gamma}^{y} \delta g^{\alpha \sigma}(x) \nabla_{\alpha}^{(x)} \nabla_{\sigma}^{(x)} G(x, y) R(y)+\int_{\Gamma}^{x} \int_{\Gamma}^{y} R(x) R(y) \delta[G(x, y)]
\end{aligned}
$$

Now we embark upon the computation of $\delta[G(x, y)]$. The Green's function in a curved background is defined in a covariant manner by (C.1c). Varying this equation with respect to metric,

$$
\begin{aligned}
\delta \square^{(x)} G(x, y)+\square^{(x)} \delta G(x, y) & =\frac{1}{2 \sqrt{g(x)}} g_{\mu \nu}(x) \delta g^{\mu \nu}(x) \delta^{2}(x-y) \\
\delta G(x, y) & =\frac{1}{2} g_{\mu \nu}(y) \delta g^{\mu \nu}(y) G(x, y)-\int_{\Gamma}^{w} G(x, w) \delta \square^{(w)} G(w, y)
\end{aligned}
$$

Here in the second line we have integrated both sides with a Green's function. The action of Laplacian on a scalar is also given by, $\square^{(x)} f(x)=\frac{1}{\sqrt{g(x)}} \partial_{\mu}\left(\sqrt{g(x)} g^{\mu \nu}(x) \partial_{\nu} f(x)\right)$. Thus the variation of the $\square$ operator is,

$$
\begin{aligned}
\delta \square^{(x)} f(x)= & \frac{1}{2} g_{\mu \nu}(x) \delta g^{\mu \nu}(x) \square^{(x)} f(x)-\frac{1}{2 \sqrt{g(x)}} \partial_{\mu}\left(\sqrt{g(x)} g_{\rho \sigma}(x) \delta g^{\rho \sigma}(x) g^{\mu \nu}(x) \partial_{\nu} f(x)\right) \\
& +\frac{1}{\sqrt{g(x)}} \partial_{\mu}\left(\sqrt{g(x)} \delta g^{\mu \nu}(x) \partial_{\nu} f(x)\right) \\
= & -\frac{1}{2} g^{\mu \nu}(x) \partial_{\nu} f(x) \nabla_{\mu}\left(g_{\rho \sigma}(x) \delta g^{\rho \sigma}(x)\right)+\frac{1}{\sqrt{g(x)}} \partial_{\mu}\left(\sqrt{g(x)} \delta g^{\mu \nu}(x) \partial_{\nu} f(x)\right) \\
= & -\frac{1}{2} g^{\mu \nu}(x) \partial_{\nu} f(x) \nabla_{\mu}\left(g_{\rho \sigma}(x) \delta g^{\rho \sigma}(x)\right)+\partial_{\mu}\left(\delta g^{\mu \nu}(x)\right) \partial_{\nu} f(x)+\delta g^{\mu \nu}(x) \partial_{\mu} \partial_{\nu} f(x) \\
& +\frac{1}{2 g(x)} \partial_{\mu}(g(x)) \delta g^{\mu \nu}(x) \partial_{\nu} f(x) \\
= & -\frac{1}{2} g^{\mu \nu}(x) \partial_{\nu} f(x) \nabla_{\mu}\left(g_{\rho \sigma}(x) \delta g^{\rho \sigma}(x)\right)+\partial_{\mu}\left(\delta g^{\mu \nu}(x)\right) \partial_{\nu} f(x)+\delta g^{\mu \nu}(x) \partial_{\mu} \partial_{\nu} f(x) \\
& +\Gamma^{\sigma}{ }_{\mu \sigma} \delta g^{\mu \nu}(x) \partial_{\nu} f(x) \\
\Rightarrow \delta \square \square^{(x)} f(x)= & -\frac{1}{2} g^{\mu \nu}(x) \partial_{\nu} f(x) \nabla_{\mu}\left(g_{\rho \sigma}(x) \delta g^{\rho \sigma}(x)\right)+\nabla_{\mu}\left(\delta g^{\mu \nu}(x)\right) \partial_{\nu} f(x)+\delta g^{\mu \nu}(x) \nabla_{\mu} \partial_{\nu} f(x)
\end{aligned}
$$

We have used chain rule of differentiation to come from the first line on r.h.s. to the second line. We have also changed the normal derivative acting on $g_{\rho \sigma}(x) \delta g^{\rho \sigma}(x)$ into a covariant derivative because it is a scalar. In the fourth line we have used the identity, $\partial_{\mu} g(x)=2 g(x) \Gamma_{\mu \nu}^{\nu}$. We have also converted some of the differentiations into covariant 
derivatives in last line. For our computations, the role of $f$ in the above computations is played by, $\int_{\Gamma}^{y} \sqrt{g(y)} R(y) G(w, y)$. Using (C.5) in (C.4) and substituting back into last term of (C.3),

$$
\begin{aligned}
\int_{\Gamma}^{x} & \int_{\Gamma}^{y} R(x) R(y) \delta[G(x, y)] \\
= & \int_{\Gamma}^{x} \int_{\Gamma}^{y} R(x) R(y)\left[\frac{1}{2} g_{\mu \nu}(y) \delta g^{\mu \nu}(y) G(x, y)-\int_{\Gamma}^{w} G(x, w) \delta \square^{(w)} G(w, y)\right] \\
= & \frac{1}{2} \int_{\Gamma}^{x} \int_{\Gamma}^{y} R(x) R(y) g_{\mu \nu}(y) \delta g^{\mu \nu}(y) G(x, y) \\
& +\frac{1}{2} \int_{\Gamma}^{x} \int_{\Gamma}^{y} \int_{\Gamma}^{w} R(x) R(y) G(x, w)\left[g^{\mu \nu}(w) \frac{\partial}{\partial w^{\nu}} G(w, y) \nabla_{\mu}^{w}\left(g_{\rho \sigma}(w) \delta g^{\rho \sigma}(w)\right)\right. \\
& \left.-2 \nabla_{\mu}^{w}\left(\delta g^{\mu \nu}(w)\right) \frac{\partial}{\partial w^{\nu}} G(w, y)-2 \delta g^{\mu \nu}(w) \nabla_{\mu}^{w} \frac{\partial}{\partial w^{\nu}} G(w, y)\right] \\
= & \frac{1}{2} \int_{\Gamma}^{x} \int_{\Gamma}^{y} R(x) R(y) g_{\mu \nu}(y) \delta g^{\mu \nu}(y) G(x, y) \\
& -\frac{1}{2} \int_{\Gamma}^{x} \int_{\Gamma}^{y} \int_{\Gamma}^{w} R(x) R(y)\left[G(x, w) \square(w) G(w, y) g_{\rho \sigma}(w) \delta g^{\rho \sigma}(w)\right] \\
& -\frac{1}{2} \int_{\Gamma}^{x} \int_{\Gamma}^{y} \int_{\Gamma}^{w} R(x) R(y)\left[g_{\rho \sigma}(w) g^{\mu \nu}(w) \frac{\partial}{\partial w^{\mu}} G(x, w) \frac{\partial}{\partial w^{\nu}} G(w, y)\right] \delta g^{\rho \sigma}(w) \\
& +\frac{1}{2} \int_{\Gamma}^{x} \int_{\Gamma}^{y} \int_{\Gamma}^{w} R(x) R(y) \nabla_{\mu}^{w}\left[G(x, w) g^{\mu \nu}(w) \frac{\partial}{\partial w^{\nu}} G(w, y) g_{\rho \sigma}(w) \delta g^{\rho \sigma}(w)\right] \\
& -\int_{\Gamma}^{x} \int_{\Gamma}^{y} \int_{\Gamma}^{w} R(x) R(y) \nabla_{\mu}^{w}\left[G(x, w) \delta g^{\mu \nu}(w) \frac{\partial}{\partial w^{\nu}} G(w, y)\right] \\
& +\int_{\Gamma}^{x} \int_{\Gamma}^{y} \int_{\Gamma}^{w} R(x) R(y)\left[\nabla_{\mu}^{w} G(x, w) \delta g^{\mu \nu}(w) \frac{\partial}{\partial w^{\nu}} G(w, y)\right] \\
& +\int_{\Gamma}^{x} \int_{\Gamma}^{y} \int_{\Gamma}^{w} R(x) R(y) G(x, w) \delta g^{\mu \nu}(w) \nabla_{\mu}^{w} \frac{\partial}{\partial w^{\nu}} G(w, y) \\
& -\int_{\Gamma}^{x} \int_{\Gamma}^{y} \int_{\Gamma}^{w} R(x) R(y) G(x, w) \delta g^{\mu \nu}(w) \nabla_{\mu}^{w} \frac{\partial}{\partial w^{\nu}} G(w, y) \\
&
\end{aligned}
$$

in the third line on r.h.s., the first two terms cancel between themselves, while the last two terms also cancel between themselves. The fourth and the fifth terms are total derivative terms that are essentially some boundary terms. These terms vanish since we are working with Dirichlet boundary conditions such that the Green's function vanishes on the boundary.

$$
\begin{aligned}
& \int_{\Gamma}^{x} \int_{\Gamma}^{y} R(x) R(y) \delta[G(x, y)] \\
& =\int_{\Gamma}^{x} \int_{\Gamma}^{y} \int_{\Gamma}^{w} R(x) R(y)\left[\frac{\partial G(w, x)}{\partial w^{\mu}} \frac{\partial G(w, y)}{\partial w^{\mu}}-\frac{1}{2} g_{\mu \nu}(w) g^{\alpha \beta}(w) \frac{\partial G(w, x)}{\partial w^{\alpha}} \frac{\partial G(w, y)}{\partial w^{\beta}}\right] \delta g^{\mu \nu}(w)
\end{aligned}
$$


Thus, the final expression of the variation of the bulk action is (with the reinstating of the overall $\frac{1}{16 \pi b^{2}}$ factor),

$$
\begin{aligned}
\delta S_{\text {cov }}^{\text {bulk }}[g]= & -\frac{1}{4 \pi b^{2}} \int_{\Gamma}^{y} \int_{\partial \Gamma}^{s} \delta \mathcal{K} R(y) G(x, y)+\frac{1}{16 \pi b^{2}} \int_{\Gamma}^{x} g_{\mu \nu}(w) \delta g^{\mu \nu}(w)(2 R(w)+8 \pi \mu) \\
& -\frac{1}{8 \pi b^{2}} \int_{\Gamma}^{x} \int_{\Gamma}^{y} \delta g^{\alpha \sigma}(w) \nabla_{\alpha}^{(w)} \nabla_{\sigma}^{(w)} G(w, y) R(y) \\
& +\frac{1}{16 \pi b^{2}} \int_{\Gamma}^{x} \int_{\Gamma}^{y} \int_{\Gamma}^{w} R(x) R(y) \\
& \times\left[\frac{\partial G(w, x)}{\partial w^{\mu}} \frac{\partial G(w, y)}{\partial w^{\mu}}-\frac{1}{2} g_{\mu \nu}(w) g^{\alpha \beta}(w) \frac{\partial G(w, x)}{\partial w^{\alpha}} \frac{\partial G(w, y)}{\partial w^{\beta}}\right] \delta g^{\mu \nu}(w)
\end{aligned}
$$

The bulk equations of motion are non local and given by:

$$
\begin{aligned}
0= & \frac{1}{16 \pi b^{2}}\left(g_{\mu \nu}(w)(2 R(w)+8 \pi \mu)+\int_{\Gamma}^{x}\left[-2 \nabla_{\mu}^{(w)} \nabla_{\nu}^{(w)} G(w, x) R(x)\right]\right. \\
& \left.+\int_{\Gamma}^{x} \int_{\Gamma}^{y}\left[\frac{\partial G(w, x)}{\partial w^{\mu}} \frac{\partial G(w, y)}{\partial w^{\mu}}-\frac{1}{2} g_{\mu \nu}(w) g^{\alpha \beta}(w) \frac{\partial G(w, x)}{\partial w^{\alpha}} \frac{\partial G(w, y)}{\partial w^{\beta}}\right] R(x) R(y)\right)
\end{aligned}
$$

Now let us look at the trace part of the equations of motion. The last term in the above equation doesn't contribute in that case.

$$
\begin{aligned}
0 & =\left(2[2 R(w)+8 \pi \mu]+\int_{\Gamma}^{x}\left[-2 \square^{(w)} G(w, x) R(x)\right]\right) \\
& =R(w)+8 \pi \mu
\end{aligned}
$$

In conformal gauge, where $g_{\mu \nu}(x)=e^{2 \phi(x)} \hat{g}_{\mu \nu}(x)$, this is same as, (3.5),

$$
\hat{R}(x)-2 \hat{\square} \phi(x)=-8 \pi \mu e^{2 \phi(x)}
$$

which is also the equation of motion for the Liouville mode $\phi$ with background metric $\hat{g}$. In $\operatorname{AdS}_{2}$ background $\left(d \hat{s}^{2}=\left(1 / \pi \mu(z+\bar{z})^{2}\right) d z d \bar{z}\right)$, the most general solution of this equation is, $[45,46]$,

$$
\phi=\frac{1}{2} \log \left[(z+\bar{z})^{2} \frac{\partial g(z) \bar{\partial} \bar{g}(\bar{z})}{(g(z)+\bar{g}(\bar{z}))^{2}}\right]
$$

where, in Euclidean space, $g(z), \bar{g}(\bar{z})$ are complex function which are complex conjugate of each other. Equivalently, in Lorentzian space, they can be chosen to be two independent real functions.

Solving (C.8) in full generality is a daunting task that we don't undertake. We show that $\mathrm{AdS}_{2}$ satisfies these equations of motion, and also provide an argument that $\mathrm{AAdS}_{2}$ geometries satisfy them too. Traceless part of (C.8) is,

$$
\begin{aligned}
0= & \int_{\Gamma}^{x}\left[-2\left(\nabla_{\mu}^{(w)} \nabla_{\nu}^{(w)} G(w, x)-\frac{1}{2} g_{\mu \nu}(w) \square^{(w)} G(w, x)\right) R(x)\right] \\
& +\int_{\Gamma}^{x} \int_{\Gamma}^{y}\left[\frac{\partial G(w, x)}{\partial w^{\mu}} \frac{\partial G(w, y)}{\partial w^{\mu}}-\frac{1}{2} g_{\mu \nu}(w) g^{\alpha \beta}(w) \frac{\partial G(w, x)}{\partial w^{\alpha}} \frac{\partial G(w, y)}{\partial w^{\beta}}\right] R(x) R(y)
\end{aligned}
$$


One way to check that $\mathrm{AdS}_{2}$ satisfies the on-shell equations of motion is to directly use the (B.9) in the above expression and do the exact computation. However, it is much easier if we think of $\mathrm{AdS}_{2}$ as Weyl scaling of flat space, $g_{\alpha \beta}^{\mathrm{AdS}}=e^{2 \Omega} \eta_{\alpha \beta}$, where for $\mathbb{H}$, $\Omega=-\log (\sqrt{\pi \mu}(z+\bar{z}))=-\log (\sqrt{4 \pi \mu} \zeta)$. We use the formula for Ricci scalar, $R(x)=$ $-2 e^{-2 \Omega} \square_{\text {flat }} \Omega(x)$ to write (C.12) as,

$$
\begin{aligned}
0= & 4 \int_{\Gamma} d^{2} x e^{2 \Omega}\left[\left(\nabla_{\mu}^{(w)} \nabla_{\nu}^{(w)} G(w, x)-\frac{1}{2} g_{\mu \nu}(w) \square^{(w)} G(w, x)\right)\left(e^{-2 \Omega} \square_{\text {flat }}^{(x)} \Omega(x)\right)\right] \\
& +4 \int_{\Gamma} d^{2} x \int_{\Gamma} d^{2} y e^{2 \Omega(x)} e^{2 \Omega(y)}\left[\frac{\partial G(w, x)}{\partial w^{\mu}} \frac{\partial G(w, y)}{\partial w^{\mu}}-\frac{1}{2} g_{\mu \nu}(w) g^{\alpha \beta}(w) \frac{\partial G(w, x)}{\partial w^{\alpha}} \frac{\partial G(w, y)}{\partial w^{\beta}}\right] \\
& \times\left(e^{-2 \Omega(x)} \square_{\text {flat }}^{(x)} \Omega(x)\right)\left(e^{-2 \Omega(y)} \square_{\text {flat }}^{(y)} \Omega(y)\right) \\
= & 4 \int_{\Gamma} d^{2} x\left[\left(\nabla_{\mu}^{(w)} \nabla_{\nu}^{(w)} \square_{\text {flat }}^{(x)} G(w, x)-\frac{1}{2} g_{\mu \nu}(w) \square^{(w)} \square_{\text {flat }}^{(x)} G(w, x)\right) \Omega(x)\right] \\
& +4 \int_{\Gamma} d^{2} x \int_{\Gamma} d^{2} y\left[\frac{\partial\left(\square_{\text {flat }}^{(x)} G(w, x)\right)}{\partial w^{\mu}} \frac{\partial\left(\square_{\text {flat }}^{(y)} G(w, y)\right)}{\partial w^{\mu}}\right. \\
& \left.-\frac{1}{2} g_{\mu \nu}(w) g^{\alpha \beta}(w) \frac{\partial\left(\square_{\text {flat }}^{(x)} G(w, x)\right)}{\partial w^{\alpha}} \frac{\partial\left(\square_{\text {flat }}^{(y)} G(w, y)\right)}{\partial w^{\beta}}\right] \times \Omega(x) \Omega(y) \\
= & 4\left[\nabla_{\mu}^{(x)} \nabla_{\nu}^{(x)} \Omega(x)-\frac{1}{2} g_{\mu \nu}(w) \square^{(w)} \Omega(x)\right]+4\left[\frac{\partial \Omega(x)}{\partial w^{\mu}} \frac{\partial \Omega(y)}{\partial w^{\mu}}-\frac{1}{2} g_{\mu \nu}(x) g^{\alpha \beta}(x) \frac{\partial \Omega(x)}{\partial x^{\alpha}} \frac{\partial \Omega(y)}{\partial x^{\beta}}\right] \\
= & 0
\end{aligned}
$$

In the second line we have used integration by parts to shift $\square_{\text {flat }}$ on the corresponding Green's functions; we have dropped the vanishing boundary terms on our way. We also use the fact discussed at the end of appendix B, that the Green's function remain unchanged for the Weyl scaled metrics, upto impositions of boundary condition. In this case the boundary condition, $G\left(\{\zeta, \tau\},\left\{0, \tau_{2}\right\}\right)=0$, is imposed by adding a contribution of a 'mirror charge' at a point reflected across the boundary. Thus the flat space Laplacian acting on this Green's function gives two $\delta$-functions, one each for the 'original charge' and 'mirror charge' ${ }^{32}$ The $\delta$-function of the mirror charge lies outside the region of integration and hence doesn't contribute.

The equations of motion (C.10), (C.12) are covariant equations under diffeomorphisms. Thus they will also be satisfied for the class of geometries that we constructed in section 4 .

We can still do slightly better and solve the equations of motion for Weyl scaled metrics around a given background. Around $\mathrm{AdS}_{2}$ background, from (C.12) we get following Virasoro constraints for $\phi$,

$$
4\left(\begin{array}{cc}
\partial^{2} \phi(z, \bar{z})-(\partial \phi(z, \bar{z}))^{2}+2 \frac{\partial \phi(z, \bar{z})}{z+\bar{z}} & 0 \\
0 & \bar{\partial}^{2} \phi(z, \bar{z})-(\bar{\partial} \phi(z, \bar{z}))^{2}+2 \frac{\bar{\partial} \phi(z, \bar{z})}{z+\bar{z}}
\end{array}\right)=0
$$

\footnotetext{
${ }^{32}$ The $\delta$-function is a flat space $\delta$-function.
} 
Solving (C.10) and (C.14) simultaneously, we get solutions of the type (C.11), but with $g, \bar{g}$ additionally restricted by the conditions,

$$
0=\left(\begin{array}{ccc}
2\left(\frac{g^{(3)}(z)}{g^{\prime}(z)}-\frac{3}{2} \frac{g^{\prime \prime}(z)^{2}}{g^{\prime}(z)^{2}}\right) & 0 \\
0 & 2\left(\frac{\bar{g}^{(3)}(z)}{\bar{g}^{\prime}(z)}-\frac{3}{2} \frac{\bar{g}^{\prime \prime}(z)^{2}}{\bar{g}^{\prime}(z)^{2}}\right)
\end{array}\right)
$$

which is basically the Schwarzian derivatives of $g(z)$ and $\bar{g}(\bar{z})$. This restricts $g(z)$ to be of the form,

$$
g(z)=\frac{a z+i b}{i c z+d}
$$

for $a, b, c, d \in \mathbb{C}$, and $\bar{g}(\bar{z})$ is its complex conjugate. Imposing the boundary condition, $g(z)+\left.\bar{g}(\bar{z})\right|_{z+\bar{z}=0}=0$ further restricts $a, b, c, d \in \mathbb{R}$. These precisely corresponds to the isometries of the geometries that we are considering. However, more general choice of these parameters gives us solutions that we call non-normalizable in this paper. These solutions diverge as $1 / \zeta$ for small deviations around identity,

$$
\begin{aligned}
a & =1+\delta a \\
b & =\delta b \\
c & =\delta c \\
d & =1-\delta a
\end{aligned}
$$

Boundary term. A similar analysis for the variation of boundary terms in (2.7) gives,

$$
\begin{aligned}
\delta S_{\mathrm{cov}}^{\mathrm{bdy}}[g]= & \frac{1}{16 \pi b^{2}} \int_{\Gamma} \delta\left(4 \sqrt{\gamma} \mathcal{K} \frac{1}{\square} R\right) \\
= & \frac{1}{4 \pi b^{2}} \int_{\Gamma}^{x} \int_{\partial \Gamma}^{s} \delta \mathcal{K}(s) G(x, s) R(x)-\frac{1}{4 \pi b^{2}} \int_{\Gamma}^{x} \int_{\partial \Gamma}^{s} \delta g^{\mu \nu}(x)\left[\nabla_{\mu}^{(x)} \nabla_{\nu}^{(x)} G(x, s) \mathcal{K}(s)\right] \\
& +\frac{1}{4 \pi b^{2}} \int_{\partial \Gamma}^{s} \int_{\Gamma}^{x} \int_{\Gamma}^{w} \delta g^{\mu \nu}(w) \\
& \times\left[\frac{\partial G(w, x)}{\partial w^{\mu}} \frac{\partial G(w, s)}{\partial w^{\mu}}-\frac{1}{2} g_{\mu \nu}(w) g^{\alpha \beta}(w) \frac{\partial G(w, x)}{\partial w^{\alpha}} \frac{\partial G(w, s)}{\partial w^{\beta}}\right] \mathcal{K}(s) R(x) \\
& -\frac{1}{2 \pi b^{2}} \int_{\partial \Gamma}^{s} \int_{\partial \Gamma}^{s^{\prime}} \delta \mathcal{K}(s) G\left(s, s^{\prime}\right) \mathcal{K}\left(s^{\prime}\right)
\end{aligned}
$$

Note that the first term in r.h.s. of (C.18) exactly cancels the last term in r.h.s. of (C.2). Moreover, the last term in (C.18) exactly cancels the variation arising from the last term in (2.7). Also in writing the above expressions we have made use of the fact that we are imposing Dirichlet boundary conditions on the metric, $\left.\delta g_{\mu \nu}\right|_{\partial \Gamma}=0$

\section{Analysing off-shell constraints}

In this section we demonstrate that the constraints coming from the traceless part of the equations of motion in the conformal gauge, viz. the 'Virasoro constraints' (3.7), do not permit any off-shell degrees of freedom apart from those representing large diffeomorphisms of $\mathrm{AdS}_{2}$ geometry. 
It is enough to carry out this analysis in absence of the large diffeomorphisms, with $\widehat{d s^{2}}$ as in (3.4). The generalization to (4.6) is obtained by applying the large diffeomorphism (4.5), in the manner explained in section 4.

Simplifying the holomorphic part of the constraints,

$$
\begin{array}{rlrl}
\partial^{2} \phi(z, \bar{z})-(\partial \phi(z, \bar{z}))^{2}+2 \frac{\partial \phi(z, \bar{z})}{z+\bar{z}} & =0 \\
\Rightarrow & \partial\left((z+\bar{z})^{2} \partial\left(e^{-\phi(z, \bar{z})}\right)\right) & =0 \\
\Rightarrow & \partial\left(e^{-\phi(z, \bar{z})}\right) & =\frac{A(\bar{z})}{(z+\bar{z})^{2}} \\
\Rightarrow & e^{-\phi(z, \bar{z})} & =-\frac{A(\bar{z})}{(z+\bar{z})}+B(\bar{z})
\end{array}
$$

Similarly, solving the anti-holomorphic part gives,

$$
e^{-\phi(z, \bar{z})}=-\frac{C(z)}{(z+\bar{z})}+D(z)
$$

In the above equations, the functions $A, B, C, D$ are arbitrary and independent, to begin with, as they appear as "constants' of integration. However, they must satisfy the requirement that the two expressions (D.2) and (D.3) for the same quantity $e^{-\phi(z, \bar{z})}$ must be (i) equal to each other and (ii) real. Assuming a general power series form of each of the functions, we find that these two requirements can only be met if $A, C$ are quadratic and $B, D$ are linear, and, in particular, of the form

$$
\begin{array}{ll}
A(\bar{z})=\mathbf{a} \bar{z}^{2}+2 i \mathbf{b} \bar{z}+\mathbf{c}, & B(\bar{z})=\mathbf{a} \bar{z}+\mathbf{d}+i \mathbf{b} \\
C(z)=\mathbf{a} z^{2}-2 i \mathbf{b} z+\mathbf{c}, & D(z)=\mathbf{a} z+\mathbf{d}-i \mathbf{b},
\end{array}
$$

leading to the following solution for the Liouville field,

$$
\begin{aligned}
e^{-\phi(z, \bar{z})} & =\frac{\mathbf{a} z \bar{z}+(\mathbf{d}+i \mathbf{b}) z+(\mathbf{d}-i \mathbf{b}) \bar{z}-\mathbf{c}}{z+\bar{z}} \\
\Rightarrow \phi(z, \bar{z}) & =\frac{1}{2} \log \left[\frac{(z+\bar{z})^{2}}{(\mathbf{a} z \bar{z}+(\mathbf{d}+i \mathbf{b}) z+(\mathbf{d}-i \mathbf{b}) \bar{z}-\mathbf{c})^{2}}\right]
\end{aligned}
$$

Here the constants $\mathbf{a}, \mathbf{b}, \mathbf{c}, \mathbf{d}$ are real. Out of these four, only three are physical. The reason is that the Virasoro constraints, expressed as in (D.1) (and the similar, antiholomorphic equation) are homogeneous linear equations in the variable $e^{-\phi(z, \bar{z})}$, which implies that $e^{-\phi(z, \bar{z})} \rightarrow$ constant $\times e^{-\phi(z, \bar{z})}$ is a symmetry of the equations. Hence, the constants $\mathbf{a}, \mathbf{b}, \mathbf{c}, \mathbf{d}$ are only determined up to a (real) scale factor.

It is important to check that the solution (D.5) of the Virasoro constraints satisfies the equation of motion (3.5). This can be done in two ways:

(i) By direct substitution of (D.5) into (3.5), we find that (3.5) is satisfied up to a term proportional to $\mathbf{a c}+(\mathbf{d}+i \mathbf{b})(\mathbf{d}-i \mathbf{b})-1$. By using the scale symmetry mentioned above, we can clearly make this vanish, e.g. by treating $\mathbf{a}, \mathbf{b}$ and $\mathbf{d}$ as independent variables and fixing $\mathbf{c}=(1-(\mathbf{d}+i \mathbf{b})(\mathbf{d}-i \mathbf{b})) / \mathbf{a}$ (this is equivalent to choosing a gauge). 
(ii) Alternatively, one can match (D.5) with the solution (3.6). We find that the parameters of the two solutions are related as follows

$$
\bar{a} c-a \bar{c}=-i \mathbf{a}, \bar{b} d-b \bar{d}=-i \mathbf{c}, \bar{b} c+a \bar{d}=\mathbf{d}-i \mathbf{b}
$$

The $\mathbb{S L}(2, \mathbb{C})$ conditions $a d+b c=1$ translate to the condition

$$
\mathbf{a c}+(\mathbf{d}+i \mathbf{b})(\mathbf{d}-i \mathbf{b})=1,
$$

As mentioned before, on this surface (D.5) solves the equation of motion (3.5). Furthermore, in the analysis of (3.6), we found that the $\mathbb{S L}(2, \mathbb{R})$ subgroup, parameterized by real values of $a, b, c, d$, correspond to trivial isometries of $\mathrm{AdS}_{2}$, and did not generate a new solution; there is a natural interpretation of this fact according to (D.6): real $a, b, c, d$ translate to $\mathbf{a}=\mathbf{b}=\mathbf{c}=0, \mathbf{d}=1$, leading to the trivial solution $\phi=0$. Thus the variables $\mathbf{a}, \mathbf{b}, \mathbf{c}, \mathbf{d}$, given by (D.6) actually parameterize the nontrivial coset $\mathbb{S L}(2, \mathbb{C}) / \mathbb{S L}(2, \mathbb{R})$. In fact, the hyperboloid (D.7) parameterizes this coset.

As mentioned before, the above analysis can be generalized to the case of the reference metric (4.6) by applying the large diffeomorphism (4.5) to the solution (D.5).

Conclusion. The Virasoro constraints completely fix the Liouville field $\phi$ (up to three real constants). As explained in the text (see section 4), the three constants need to be fixed as boundary conditions for the path integral (since they correspond to non-normalizable deformations). Thus, there are no off-shell variables (i.e. variables appearing in the path integration) that come from the Liouville field $\phi$. The only off-shell variables are represented by the large diffeomorphisms as in (4.9).

\section{E Exact computation of asymptotic $\mathrm{AdS}_{2}$ geometries}

We use the knowledge of exact asymptotically $\mathrm{AdS}_{3}$ geometries to construct $\mathrm{AAdS}_{2}$ geometries. In $\mathrm{AdS}_{3}$ the space of solutions of spacetimes with constant negative curvature is given by, [51, 52],

$$
d s^{2}=L_{\left(\mathrm{AdS}_{3}\right)}^{2}\left(\frac{d \zeta^{2}+2 d x d \bar{x}}{\zeta^{2}}+L(x) d x^{2}+\bar{L}(\bar{x}) d \bar{x}^{2}-\frac{\zeta^{2}}{2} L(x) \bar{L}(\bar{x}) d x d \bar{x}\right)
$$

where, $L(x), \bar{L}(\bar{x})$ are holomorphic and anti-holomorphic functions, and related to the holographic stress tensor, [59]. In the above references it is discussed how following large diffeomorphisms generate the above class of geometries from the Poincare $\mathrm{AdS}_{3}$ geometry $\left(d s^{2}=L_{\left(\operatorname{AdS}_{3}\right)}^{2}\left(d u^{2}+2 d y d \bar{y}\right) / u^{2}\right)$,

$$
\begin{aligned}
& y=f(x)+\frac{2 \zeta^{2} f^{\prime}(x)^{2} \bar{f}^{\prime \prime}(\bar{x})}{8 f^{\prime}(x) \bar{f}^{\prime}(\bar{x})-\zeta^{2} f^{\prime \prime}(x) \bar{f}^{\prime \prime}(\bar{x})} \\
& y=\bar{f}(\bar{x})+\frac{2 \bar{\zeta}^{2} \bar{f}^{\prime}(\bar{x})^{2} f^{\prime \prime}(x)}{8 f^{\prime}(x) \bar{f}^{\prime}(\bar{x})-\zeta^{2} f^{\prime \prime}(x) \bar{f}^{\prime \prime}(\bar{x})} \\
& u=\zeta \frac{\left(4 f^{\prime}(x) \bar{f}^{\prime}(\bar{x})\right)^{3 / 2}}{8 f^{\prime}(x) \bar{f}^{\prime}(\bar{x})-\zeta^{2} f^{\prime \prime}(x) \bar{f}^{\prime \prime}(\bar{x})}
\end{aligned}
$$


In general, in any arbitrary dimensions, it is not difficult to solve for the asymptotic Killing vectors for any spacetime. The special feature of $\mathrm{AdS}_{3}$ is the fact that these infinitesimal diffeomorphisms can be integrated to non-linear order. $\mathrm{AdS}_{2}$ being a more constrained geometry, also enjoys this same feature. Here, we use the known results of the exact non-linear diffeomorphisms in $\mathrm{AdS}_{3}$ to construct the class of asymptotic $\mathrm{AdS}_{2}$ solutions. In Cartesian coordinates, $y=(x+i \tau) / \sqrt{2}, \bar{y}=(x-i \tau) / \sqrt{2}$, one can obtain $\mathrm{AdS}_{2}$ as a reduction of $\mathrm{AdS}_{3}$ b y restricting to $x=0$ slice. Restricting ourselves to those transformations that keeps this $\mathrm{AdS}_{2}$ slice invariant, i.e., for $f(x)+\left.\bar{f}(\bar{x})\right|_{x+\bar{x}=0}=0$, we find that the following coordinate transformations are precisely those which generate large diffeomorphisms in $\mathrm{AdS}_{2}$, (4.5), while keeping us within Fefferman-Graham gauge,

$$
\tilde{\tau}=f(\tau)-\frac{2 \zeta^{2} f^{\prime \prime}(\tau) f^{\prime}(\tau)^{2}}{4 f^{\prime}(\tau)^{2}+\zeta^{2} f^{\prime \prime}(\tau)^{2}}, \quad \tilde{\zeta}=\frac{4 \zeta f^{\prime}(\tau)^{3}}{4 f^{\prime}(\tau)^{2}+\zeta^{2} f^{\prime \prime}(\tau)^{2}}
$$

These transformations map the $\mathrm{AdS}_{2}$ metrics, $d s^{2}=\left(d \tilde{\zeta}^{2}+d \tilde{\tau}^{2}\right) /\left(4 \pi \mu \tilde{\zeta}^{2}\right)$ to $\mathrm{AAdS}_{2}$ geometries, $d s^{2}=\left(d \zeta^{2}+\left(1-\frac{\zeta^{2}}{2}\{f(\tau), \tau\}\right)^{2} d \tau^{2}\right) /\left(4 \pi \mu \zeta^{2}\right)$.

\section{F Quantum corrections to the classical action}

In this section we discuss the issue of gauge fixing the action (2.7). The idea and FaddeevPopov procedure to arrive at the same: we introduce a functional delta-function in our path integral using the Faddeev-Popov prescription. The corresponding determinant is then written in terms of fermionic ghosts, which gives rise to new ghost-graviton interaction vertices. ${ }^{33}$

The Faddeev-Popov determinant is defined in terms of the gauge-fixing $\delta$-function as follows,

$$
\begin{aligned}
1= & \Delta_{\mathrm{FP}}[\hat{g}[f(\tau)], \phi] \times \int\left[\mathcal{D} \epsilon^{(s)}\right][\mathcal{D} \phi][\mathcal{D} f(\tau)] \delta\left(g^{\epsilon^{(s)}}-e^{2 \phi} \hat{g}[f(\tau)]\right) \\
& \times \delta\left(\epsilon^{(s)}\left(z_{1}\right)\right) \delta\left(\epsilon^{(s)}\left(z_{2}\right)\right) \delta\left(\epsilon^{(s)}\left(z_{3}\right)\right)
\end{aligned}
$$

Here, we are denoting the small diffeomorphisms (these are the gauge-symmetry of the theory) by $\epsilon^{(s)}$. In the subsequent discussion we will drop the $(s)$ superscript to conciseness. $\phi$ denotes the Weyl degree of freedom and will eventually become the Liouville mode. Since our theory is not Weyl-invariant, unlike in String theory, we don't factor out these degrees of freedom. Finally, $f(\tau)$ denotes the degree of freedom due to large diffeomorphisms that is discussed in section 4. We are gauge fixing (using only small diffeomorphisms) an arbitrary metric to a metric that is Weyl equivalent to metrics (4.6). This procedure will give us the Jacobian corresponding to change of integration 'variable' from $[\mathcal{D} g]$ to $[\mathcal{D} \phi][\mathcal{D} f(\tau)]$. The $\delta$-functions have been included in the above expression to fix the residual gauge symmetry corresponding to our gauge choice. This is precisely the $\mathbb{S L}(2, \mathbb{R})$ isometry of the geometries (4.6), and hence we choose to fix three arbitrary points in the interior

\footnotetext{
${ }^{33}$ In the subsequent discussion in this particular appendix, we call the $f$ degree of freedom of section 4 corresponding to large diffeomorphisms of $\mathrm{AdS}_{2}$ as 'gravitons'.
} 
of $\mathrm{AAdS}_{2}$ geometries. The path integral that we are interested in computing is formally written as,

$$
Z=\int \frac{[\mathcal{D} g]}{V_{\epsilon}} e^{-S[g]}, \quad V_{\epsilon} \text { is the volume of the symmetry group }
$$

and inserting (F.1) into this path integral, we get,

$$
\begin{aligned}
Z & =\int \frac{[\mathcal{D} g][\mathcal{D} \epsilon][\mathcal{D} \phi][\mathcal{D} f(\tau)]}{V_{\epsilon}} \times \Delta_{\mathrm{FP}}[\hat{g}[f(\tau)], \phi] \delta\left(g^{\epsilon}-e^{2 \phi} \hat{g}[f(\tau)]\right) \times e^{-S[g]} \times(\delta \text {-functions }) \\
& =\int \frac{[\mathcal{D} \tilde{g}][\mathcal{D} \epsilon][\mathcal{D} \phi][\mathcal{D} f(\tau)]}{V_{\epsilon}} \times \Delta_{\mathrm{FP}}[\hat{g}[f(\tau)], \phi] \delta\left(\tilde{g}-e^{2 \phi} \hat{g}[f(\tau)]\right) \times e^{-S[\tilde{g}]} \times(\delta \text {-functions }) \\
& =\int \frac{[\mathcal{D} \epsilon][\mathcal{D} \phi][\mathcal{D} f(\tau)]}{V_{\epsilon}} \times \Delta_{\mathrm{FP}}[\hat{g}[f(\tau)], \phi] \times e^{-S\left[e^{2 \phi} \hat{g}[f(\tau)]\right]} \times(\delta \text {-functions }) \\
& =\int[\mathcal{D} \phi][\mathcal{D} f(\tau)] \times \Delta_{\mathrm{FP}}[\hat{g}[f(\tau)], \phi] \times e^{-S\left[e^{2 \phi} \hat{g}[f(\tau)]\right]} \times(\delta \text {-functions })
\end{aligned}
$$

In the second line on r.h.s., we have changed integration 'variables' from $\mathcal{D} g$ to $\mathcal{D} \tilde{g}$, where, $g=: \tilde{g}^{\epsilon^{-1}}$ and used the fact that action and measure are both gauge invariant. In the third line we have integrated over the metric degrees of freedom using the $\delta$-function. In the last line we have used the fact that the integrand of third line doesn't depend on $\epsilon$ anymore, integration over which simply gives us the volume of the symmetry group.

Faddeev Popov determinant can be easily written in terms of the $b$ and $c$ ghosts as,

$$
\begin{aligned}
\Delta_{\mathrm{FP}}[\hat{g}[f(\tau)], \phi]= & \int \mathcal{D} c_{\alpha} \mathcal{D} b^{\alpha \beta} \mathcal{D} \mathfrak{f}_{\alpha} \exp \left[-\left(b^{\alpha \beta}(\hat{P} c)_{\alpha \beta}-b^{\alpha \beta}(\hat{P} \mathfrak{f})_{\alpha \beta}\right)\right] \\
& \times\left[\frac{c\left(z_{1}\right) c\left(z_{2}\right) c\left(z_{3}\right)}{\left(z_{1}-z_{2}\right)\left(z_{2}-z_{3}\right)\left(z_{3}-z_{1}\right)}\right]
\end{aligned}
$$

here, $c$-insertions are equivalent to the $\delta$-functions appearing in the previous expressions. $b^{\alpha \beta}$ is a symmetric-traceless tensor, and thus has only 2 degrees of freedom. We have defined operator $\hat{P}$ such that,

$$
(\hat{P} x)_{\alpha \beta}:={ }^{(f)} \nabla_{(\alpha} x_{\beta)}-\left({ }^{(f)} \nabla \cdot x\right) \hat{g}[f(\tau)]_{\alpha \beta}
$$

${ }^{(f)} \nabla$ is the covariant derivative w.r.t. geometries in (4.6). $\mathfrak{f}$ is defined in terms of the fermionized large diffeomorphisms (4.4) as,

$$
\mathfrak{f}=\left(\mathfrak{k}(\tau)^{\zeta}-\frac{\mathfrak{k}^{\prime}\left(\mathcal{( \tau )}^{2}\right.}{2} \mathfrak{k}^{\prime \prime \prime}(\tau)\right)
$$

where again, $\mathfrak{k}$ are the fermionized ghost counter-part of the field appearing in (4.4). The above action can be expanded and written in terms of the components:

$$
\hat{P} \mathfrak{f}=\left(\begin{array}{cc}
\frac{1}{\left(\{f(\tau), \tau\} \zeta^{2}-2\right)^{3}}\left[-2 \zeta^{4} \partial_{\tau}(\{f(\tau), \tau\}) \mathfrak{k}^{\prime \prime}(\tau)\right. & \frac{\left(\zeta^{2}\{f(\tau), \tau\}+2\right)\left(\zeta^{2} \mathfrak{k}^{\prime \prime}(\tau)-2 \mathfrak{k}(\tau)\right)}{\zeta\left(\zeta^{2}\{f(\tau), \tau\}-2\right)} \\
+4 \zeta^{2} \partial_{\tau}(\{f(\tau), \tau\}) \mathfrak{k}(\tau)+2 \zeta^{2}\left(\{f(\tau), \tau\} \zeta^{2}-2\right) \mathfrak{k}^{(3)}(\tau) & \\
\left.+\left(\{f(\tau), \tau\} \zeta^{2}-2\right)\left(\{f(\tau), \tau\}\left(\{f(\tau), \tau\} \zeta^{2}-8\right) \zeta^{2}+8\right) \mathfrak{k}^{\prime}(\tau)\right] & \\
\frac{\left(\zeta^{2}\{f(\tau), \tau\}+2\right)\left(\zeta^{2} \mathfrak{k}^{\prime \prime}(\tau)-2 \mathfrak{k}(\tau)\right)}{\zeta\left(\zeta^{2}\{f(\tau), \tau\}-2\right)} & \frac{1}{4\{f(\tau), \tau\} \zeta^{2}-8}\left[16 \mathfrak{k}^{\prime}(\tau)+2 \zeta^{4} \partial_{\tau}(\{f(\tau), \tau\}) \mathfrak{k}^{\prime \prime}(\tau)\right. \\
& -\zeta^{2}\{f(\tau), \tau\}\left(\{f(\tau), \tau\} \zeta^{2}-6\right)\left(\{f(\tau), \tau\} \zeta^{2}-4\right) \mathfrak{k}^{\prime}(\tau) \\
& \left.-4 \zeta^{2} \partial_{\tau}(\{f(\tau), \tau\}) \mathfrak{k}(\tau)-2 \zeta^{2}\left(\{f(\tau), \tau\} \zeta^{2}-2\right) \mathfrak{k}^{(3)}(\tau)\right]
\end{array}\right)
$$




$$
\hat{P} c=\left(\begin{array}{cc}
\frac{1}{\zeta\left(\{f(\tau), \tau\} \zeta^{2}-2\right)^{3}}\left[4 \zeta^{3} \partial_{\tau}(\{f(\tau), \tau\}) c_{\tau}(\zeta, \tau)\right. & \left(c_{\zeta}^{(0,1)}(\zeta, \tau)+c_{\tau}^{(1,0)}(\zeta, \tau)\right) \\
-4 \zeta\left(\{f(\tau), \tau\} \zeta^{2}-2\right) c_{\tau}^{(0,1)}(\zeta, \tau) & -2 \frac{\left.\zeta^{2}\{f(\tau), \tau\}+2\right) c_{\tau}(\zeta, \tau)}{\zeta\left(\zeta^{2}\{f(\tau), \tau\}-2\right)} \\
+\zeta\left(\{f(\tau), \tau\} \zeta^{2}-2\right)^{3} c_{\zeta}^{(1,0)}(\zeta, \tau) & \\
\left.-4\left(\{f(\tau), \tau\} \zeta^{2}-2\right)^{2} c_{\zeta}(\zeta, \tau)\right] & \frac{1}{} \\
& \frac{1}{4 \zeta\left(\{f(\tau), \tau\} \zeta^{2}-2\right)}\left[-4 \zeta^{3} \partial_{\tau}(\{f(\tau), \tau\}) c_{\tau}(\zeta, \tau)\right. \\
\left(c_{\zeta}^{(0,1)}(\zeta, \tau)+c_{\tau}^{(1,0)}(\zeta, \tau)\right) & +4 \zeta\left(\{f(\tau), \tau\} \zeta^{2}-2\right) c_{\tau}^{(0,1)}(\zeta, \tau) \\
-2 \frac{\left(\zeta^{2}\{f(\tau), \tau\}+2\right) c_{\tau}(\zeta, \tau)}{\zeta\left(\zeta^{2}\{f(\tau), \tau\}-2\right)} & +4\left(\{f(\tau), \tau\} \zeta^{2}-2\right)^{2} c_{\zeta}(\zeta, \tau) \\
& \left.-\zeta\left(\{f(\tau), \tau\} \zeta^{2}-2\right)^{3} c_{\zeta}^{(1,0)}(\zeta, \tau)\right]
\end{array}\right)
$$

\section{G Weyl anomaly in manifolds with boundary}

In this section we compute the most general boundary term for Weyl anomaly in 2dimensions on a manifold with a boundary allowed by the Wess-Zumino consistency condition. Let us start with the variation of (2.7) under a Weyl transformation,

$$
\begin{aligned}
& \delta_{W} S_{\mathrm{cov}} \\
& =-\frac{1}{4 \pi b^{2}} \int_{\Gamma} \sqrt{g}(R+8 \pi \mu) \delta \omega+\left.\frac{1}{4 \pi b^{2}} \int_{\partial \Gamma} \sqrt{\gamma(s)} \int_{\Gamma} \sqrt{g(x)} \delta \omega(s) R(x) \hat{n}^{\mu}(s) \frac{\partial}{\partial y^{\mu}} G(x, y)\right|_{y=s} \\
& +\left.\frac{1}{2 \pi b^{2}} \int_{\partial \Gamma} \sqrt{\gamma\left(s_{1}\right)} \int_{\partial \Gamma} \sqrt{\gamma\left(s_{2}\right)} \delta \omega\left(s_{2}\right) \mathcal{K}\left(s_{1}\right) \hat{n}^{\mu}\left(s_{2}\right) \frac{\partial}{\partial y^{\mu}} G\left(s_{1}, y\right)\right|_{y=s_{2}}-\frac{1}{2 \pi b^{2}} \int_{\partial \Gamma} \sqrt{\gamma(s)} \mathcal{K}(s) \delta \omega(s)
\end{aligned}
$$

Under a second Weyl transformation

$$
\begin{aligned}
\delta_{W_{2}}\left(\delta_{W_{1}} S_{\text {cov }}\right)= & -\frac{1}{2 \pi b^{2}} \int_{\partial \Gamma} \sqrt{\gamma(s)} \partial^{\mu} \delta \omega(s) \partial_{\mu} \delta \omega_{2}(s)-\frac{4 \mu}{b^{2}} \int_{\Gamma} \sqrt{g} \delta \omega_{2}(x) \delta \omega_{1}(x) \\
& +\frac{1}{2 \pi b^{2}} \int_{\partial \Gamma} \sqrt{\gamma\left(s_{1}\right)} \int_{\partial \Gamma} \sqrt{\gamma\left(s_{2}\right)} \delta \omega_{1}\left(s_{1}\right) \delta \omega_{2}\left(s_{2}\right) \hat{n}^{\mu}\left(s_{1}\right) \hat{n}^{\nu}\left(s_{2}\right) \partial_{\mu} \partial_{\nu} G\left(s_{1}, s_{2}\right)
\end{aligned}
$$

therefore, since all the terms in the above equation are symmetric in $\delta \omega_{1}$ and $\delta \omega_{2}$, we have,

$$
\delta_{W_{2}}\left(\delta_{W_{1}} S_{\mathrm{cov}}\right)-\delta_{W_{1}}\left(\delta_{W_{2}} S_{\mathrm{cov}}\right)=0
$$

Thus the boundary terms that we have introduced are consistent with the Wess-Zumino conditions. All the boundary terms that we have introduced are consistent with the general analysis in [60]. 
Open Access. This article is distributed under the terms of the Creative Commons Attribution License (CC-BY 4.0), which permits any use, distribution and reproduction in any medium, provided the original author(s) and source are credited.

\section{References}

[1] A. Kitaev, A simple model of quantum holography, talks at KITP, 7 April and 27 May 2015.

[2] S. Sachdev and J. Ye, Gapless spin fluid ground state in a random, quantum Heisenberg magnet, Phys. Rev. Lett. 70 (1993) 3339 [cond-mat/9212030] [INSPIRE].

[3] S. Sachdev, Holographic metals and the fractionalized Fermi liquid, Phys. Rev. Lett. 105 (2010) 151602 [arXiv:1006.3794] [INSPIRE].

[4] S. Sachdev, Bekenstein-Hawking Entropy and Strange Metals, Phys. Rev. X 5 (2015) 041025 [arXiv: 1506.05111] [INSPIRE].

[5] J. Maldacena and D. Stanford, Remarks on the Sachdev-Ye-Kitaev model, Phys. Rev. D 94 (2016) 106002 [arXiv:1604.07818] [INSPIRE].

[6] R. Gurau, The complete $1 / N$ expansion of a $S Y K$-like tensor model, Nucl. Phys. B 916 (2017) 386 [arXiv:1611.04032] [InSPIRE].

[7] E. Witten, An SYK-Like Model Without Disorder, arXiv:1610.09758 [INSPIRE].

[8] I.R. Klebanov and G. Tarnopolsky, Uncolored random tensors, melon diagrams and the Sachdev-Ye-Kitaev models, Phys. Rev. D 95 (2017) 046004 [arXiv:1611.08915] [InSPIRE].

[9] R. Gurau, Colored Group Field Theory, Commun. Math. Phys. 304 (2011) 69 [arXiv:0907.2582] [INSPIRE].

[10] Y. Gu, X.-L. Qi and D. Stanford, Local criticality, diffusion and chaos in generalized Sachdev-Ye-Kitaev models, JHEP 05 (2017) 125 [arXiv: 1609.07832] [INSPIRE].

[11] D.J. Gross and V. Rosenhaus, A Generalization of Sachdev-Ye-Kitaev, JHEP 02 (2017) 093 [arXiv: 1610.01569] [INSPIRE].

[12] M. Berkooz, P. Narayan, M. Rozali and J. Simón, Higher Dimensional Generalizations of the SYK Model, JHEP 01 (2017) 138 [arXiv:1610.02422] [INSPIRE].

[13] J. Engelsöy, T.G. Mertens and H. Verlinde, An investigation of $A d S_{2}$ backreaction and holography, JHEP 07 (2016) 139 [arXiv:1606.03438] [INSPIRE].

[14] G. Turiaci and H. Verlinde, On CFT and Quantum Chaos, JHEP 12 (2016) 110 [arXiv: 1603.03020] [INSPIRE].

[15] A. Jevicki and K. Suzuki, Bi-Local Holography in the SYK Model: Perturbations, JHEP 11 (2016) 046 [arXiv: 1608.07567] [INSPIRE].

[16] A. Jevicki, K. Suzuki and J. Yoon, Bi-Local Holography in the SYK Model, JHEP 07 (2016) 007 [arXiv: 1603.06246] [INSPIRE].

[17] W. Fu, D. Gaiotto, J. Maldacena and S. Sachdev, Supersymmetric Sachdev-Ye-Kitaev models, Phys. Rev. D 95 (2017) 026009 [arXiv:1610.08917] [INSPIRE].

[18] J.S. Cotler et al., Black Holes and Random Matrices, JHEP 05 (2017) 118 [arXiv: 1611.04650] [INSPIRE].

[19] A.M. García-García and J.J.M. Verbaarschot, Spectral and thermodynamic properties of the Sachdev-Ye-Kitaev model, Phys. Rev. D 94 (2016) 126010 [arXiv:1610.03816] [INSPIRE]. 
[20] R.A. Davison, W. Fu, A. Georges, Y. Gu, K. Jensen and S. Sachdev, Thermoelectric transport in disordered metals without quasiparticles: The Sachdev-Ye-Kitaev models and holography, Phys. Rev. B 95 (2017) 155131 [arXiv:1612.00849] [INSPIRE].

[21] C. Krishnan, S. Sanyal and P.N. Bala Subramanian, Quantum Chaos and Holographic Tensor Models, JHEP 03 (2017) 056 [arXiv: 1612.06330] [INSPIRE].

[22] T. Li, J. Liu, Y. Xin and Y. Zhou, Supersymmetric SYK model and random matrix theory, JHEP 06 (2017) 111 [arXiv: 1702.01738] [INSPIRE].

[23] M. Cvetič and I. Papadimitriou, AdS $S_{2}$ holographic dictionary, JHEP 12 (2016) 008 [Erratum ibid. 01 (2017) 120] [arXiv:1608.07018] [INSPIRE].

[24] J. Maldacena, D. Stanford and Z. Yang, Conformal symmetry and its breaking in two dimensional Nearly Anti-de-Sitter space, PTEP 2016 (2016) 12C104 [arXiv:1606.01857] [INSPIRE].

[25] A. Almheiri and J. Polchinski, Models of $A d S_{2}$ backreaction and holography, JHEP 11 (2015) 014 [arXiv: 1402.6334] [INSPIRE].

[26] J. Maldacena, S.H. Shenker and D. Stanford, A bound on chaos, JHEP 08 (2016) 106 [arXiv: 1503.01409] [INSPIRE].

[27] K. Jensen, Chaos in AdS $S_{2}$ Holography, Phys. Rev. Lett. 117 (2016) 111601 [arXiv: 1605.06098] [INSPIRE].

[28] A. Alekseev and S.L. Shatashvili, Path Integral Quantization of the Coadjoint Orbits of the Virasoro Group and 2D Gravity, Nucl. Phys. B 323 (1989) 719 [inSPIRE].

[29] B. Rai and V.G.J. Rodgers, From Coadjoint Orbits to Scale Invariant WZNW Type Actions and 2-D Quantum Gravity Action, Nucl. Phys. B 341 (1990) 119 [InSPIRE].

[30] A.A. Kirillov, Elements of the representation theory, Nauka, Moscow (1972).

[31] A.M. Polyakov, Quantum Gravity in Two-Dimensions, Mod. Phys. Lett. A 2 (1987) 893 [INSPIRE].

[32] A. Dhar, G. Mandal and S.R. Wadia, Nonrelativistic fermions, coadjoint orbits of $W$ (infinity) and string field theory at $c=1$, Mod. Phys. Lett. A 7 (1992) 3129 [hep-th/9207011] [INSPIRE].

[33] A. Dhar, G. Mandal and S.R. Wadia, Classical Fermi fluid and geometric action for $c=1$, Int. J. Mod. Phys. A 8 (1993) 325 [hep-th/9204028] [INSPIRE].

[34] S.R. Das, A. Dhar, G. Mandal and S.R. Wadia, Gauge theory formulation of the $C=1$ matrix model: Symmetries and discrete states, Int. J. Mod. Phys. A 7 (1992) 5165 [hep-th/9110021] [INSPIRE].

[35] B. Balthazar, V.A. Rodriguez and X. Yin, The c=1 String Theory S-matrix Revisited, arXiv: 1705.07151 [INSPIRE].

[36] G. Mandal, Fermions from half-BPS supergravity, JHEP 08 (2005) 052 [hep-th/0502104] [INSPIRE].

[37] H. Lin, O. Lunin and J.M. Maldacena, Bubbling AdS space and 1/2 BPS geometries, JHEP 10 (2004) 025 [hep-th/0409174] [INSPIRE].

[38] E. Witten, Coadjoint Orbits of the Virasoro Group, Commun. Math. Phys. 114 (1988) 1 [INSPIRE].

[39] S. Weinberg, The quantum theory of fields. Vol. 2: Modern applications, Cambridge University Press (2013). 
[40] L. Susskind and E. Witten, The Holographic bound in anti-de Sitter space, hep-th/9805114 [INSPIRE].

[41] T. Faulkner, H. Liu and M. Rangamani, Integrating out geometry: Holographic Wilsonian $R G$ and the membrane paradigm, JHEP 08 (2011) 051 [arXiv:1010.4036] [INSPIRE].

[42] I. Heemskerk and J. Polchinski, Holographic and Wilsonian Renormalization Groups, JHEP 06 (2011) 031 [arXiv:1010.1264] [INSPIRE].

[43] G. Mandal and P. Nayak, Revisiting AdS/CFT at a finite radial cut-off, JHEP 12 (2016) 125 [arXiv: 1608.00411] [INSPIRE].

[44] A. Maloney and E. Witten, Quantum Gravity Partition Functions in Three Dimensions, JHEP 02 (2010) 029 [arXiv:0712.0155] [INSPIRE].

[45] N. Seiberg, Notes on quantum Liouville theory and quantum gravity, Prog. Theor. Phys. Suppl. 102 (1990) 319 [INSPIRE].

[46] E. Witten, Some Exact Multi-Instanton Solutions of Classical Yang-Mills Theory, Phys. Rev. Lett. 38 (1977) 121 [INSPIRE].

[47] D. Bagrets, A. Altland and A. Kamenev, Sachdev-Ye-Kitaev model as Liouville quantum mechanics, Nucl. Phys. B 911 (2016) 191 [arXiv:1607.00694] [InSPIRE].

[48] M. Hotta, Asymptotic isometry and two-dimensional anti-de Sitter gravity, gr-qc/9809035 [INSPIRE].

[49] M. Cadoni and S. Mignemi, Asymptotic symmetries of $A d S_{2}$ and conformal group in $D=1$, Nucl. Phys. B 557 (1999) 165 [hep-th/9902040] [INSPIRE].

[50] J.D. Brown and M. Henneaux, Central Charges in the Canonical Realization of Asymptotic Symmetries: An Example from Three-Dimensional Gravity, Commun. Math. Phys. 104 (1986) 207 [InSPIRE].

[51] M. Bañados, Three-dimensional quantum geometry and black holes, hep-th/9901148 [INSPIRE].

[52] M.M. Roberts, Time evolution of entanglement entropy from a pulse, JHEP 12 (2012) 027 [arXiv: 1204.1982] [INSPIRE].

[53] R. Nakayama and T. Suzuki, Study of the $A d S_{2} / C F T_{1}$ Correspondence with the Contribution from the Weyl Anomaly, PTEP 2017 (2017) 083B06 [arXiv:1701.08518] [INSPIRE].

[54] R. Osserman, A new variant of the Schwarz-Pick-Ahlfors lemma, math/9803158.

[55] S.W. Hawking and D.N. Page, Thermodynamics of Black Holes in anti-de Sitter Space, Commun. Math. Phys. 87 (1983) 577 [INSPIRE].

[56] J. Polchinski and V. Rosenhaus, The Spectrum in the Sachdev-Ye-Kitaev Model, JHEP 04 (2016) 001 [arXiv:1601.06768] [INSPIRE].

[57] G. Mandal, Coadjoint Orbits and Liouville Bulk Dual, talk at String Theory: Past and Present, ICTS-TIFR, Bangalore, 11-13 January 2017 [https://www.youtube.com/watch?v=x7_Jx31BtVU].

[58] D. Borthwick, Spectral Theory of Infinite-Area Hyperbolic Surfaces, Birkhauser (1987).

[59] V. Balasubramanian and P. Kraus, A Stress tensor for Anti-de Sitter gravity, Commun. Math. Phys. 208 (1999) 413 [hep-th/9902121] [INSPIRE].

[60] J. Polchinski, String Theory, Volume I, Cambridge University Press (1998). 